\title{
The Chemistry of the Noncrystalline State
}

\author{
By Stephen R. Elliott, C. N. R. Rao, and John M. Thomas*
}

This article sets out to describe and account for the chemical and physical consequences of the presence of gross disorder in solids. Knowledge of the structure of such disordered materials is an obvious prerequisite to a further understanding of other properties and behavior, and our current knowledge of the structure of various noncrystalline systems is discussed together with the experimental techniques which need to be employed in order to obtain such information. The so-called glass transition, which takes place as a liquid is supercooled below the crystallization temperature, is discussed in terms of the various models which have been proposed to account for this phenomenon. The effect of noncrystallinity on electronic properties is also discussed, and we highlight new developments in the understanding of electron localization and transport processes. Finally, two applications of amorphous solids are considered in some detail: optical fibers for use in communication networks and "superionic" glasses for possible use in solid-state batteries.

\section{Introduction}

Many catalysts and catalyst supports, most optical fibers and numerous other optical components, several xerographic photoreceptors and thin-film transistors, large-area solar cells, a substantial proportion of biominerals-these and some other materials of considerable technological and biological importance have one feature in common: they are noncrystalline. Chemists are so innured to the pictures portrayed in textbooks of elemental structural chemistry that they are apt to assume that all solids are devoid of structural irregularity. There is a subconscious tendency to believe that solid materials are composed of unit cells which are regularly stacked or properly packed in a threedimensionally repeated fashion. But even crystalline solids contain defects ranging in character from those of a point nature (where there are vacancies or interstitial species), to line defects (dislocations) through to sub-grain boundaries (i.e. intersecting or aligned dislocations) which separate microcrystallites of one orientation from adjacent ones of another. Chemists have known for a long time, however, how to produce solids which are noncrystalline even though they may have been ignorant of the structural niceties of such solids - silica glass is an obvious example, but even liquids such as isopentane when cooled to $65 \mathrm{~K}$ and ethanol cooled to $90 \mathrm{~K}$ form a glass (as anyone who has looked into ways of establishing the validity of the third law of thermodynamics knows), and so do very many other materials. Even metals and alloys, if quenched sufficiently rapidly to low temperatures, can form noncrystalline, isotropic solids.

Noncrystalline solids possess no long-range order (i.e. no translational periodicity-see Fig. 1). Their structure can be viewed as being similar to that of a frozen liquid, but with the thermal fluctuations present in a liquid frozen

[*] Prof. J. M. Thomas, Dr. S. R. Elliott

Department of Physical Chemistry, University of Cambridge

Lensfield Road, Cambridge CB2 IEP (UK)

Prof. C. N. R. Rao

Solid-State and Structural Chemistry Unit

Indian Institute of Science

Bangalore 560012 (India) out leaving only "static" structural disorder. As a result, $\mathrm{X}$-ray diffraction patterns consisting of sharp Bragg spots or rings, characteristic of the periodicity (long-range order) in single crystals or polycrystalline aggregates, respectively, become broadened into diffuse haloes. The same effect is observed of course also for very small microcrystallites (having diameters less than $\simeq 50 \AA$ ), and as such sometimes it is difficult, by diffraction means alone, to determine whether an "X-ray amorphous" material is truly amorphous or just grossly disordered. In certain cases, however, e.g. in the case of Si or Ge, a Bragg peak characteristic of a particular interatomic spacing in the crystal is completely absent in the amorphous phase, ${ }^{[1]}$ and so in such cases there is little or no ambiguity. The atoms in a glass, however, are seldom distributed completely at random, as the simple picture might imply, but rather there is often present a considerable degree of local structural or chemical order. The extent of the structural "order" within an amorphous or noncrystalline solid (we use the terms a)
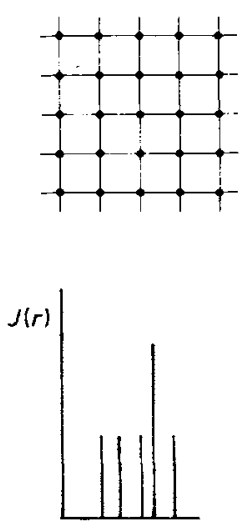

b)

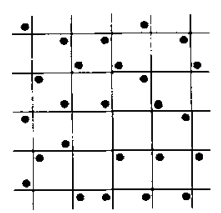

c)

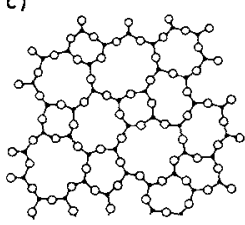

Fig. 1. Types of disorder. (a) Ordered lattice; (b) vibrational disorder; (c) topological disorder. The radial distribution functions (RDFs), $J(r)$, describing such two-dimensional structures are shown below each figure. Note that the widths of all peaks are equal in the RDF of a lattice with just vibrational disorder, whereas the peaks become progressively wider with increasing distance for a topologically disordered structure as a result of static disorder (bond angle fuctuations etc.). 
synonymously) is the subject of much current debate and we return to it later in Sections 4 and 5 .

A recent exciting development has been the discovery ${ }^{[2]}$ of long-range orientational order accompanied by fivefold point-group symmetry in small regions of rapidly quenched specimens of the alloy $\mathrm{Al}_{86} \mathrm{Mn}_{14}$; tenfold symmetry axes were observed in the patterns of sharp Bragg spots produced by electron diffraction for certain orientations of the specimens. The importance of such observations lies in the fact that classical crystallography precludes the presence of fivefold or tenfold symmetry axes for truly periodic crystals, i.e. those having long-range translational order. Spurious tenfold symmetry in diffraction patterns can be produced by twinning of ordinary crystals, but lattice-imaging micrographs of the $\mathrm{Al}-\mathrm{Mn}$ alloys clearly reveal the existence of five coexisting sets of atomic planes with normals pointing to the vertices of a regular decagon. Thus, the fivefold symmetry appears to be an intrinsic property, and the structures containing such symmetry elements have been termed "quasi-crystals". ${ }^{[3]}$ Although several theories have been proposed to account for quasi-crystals (see ref. [4] for a review), none at present are entirely satisfactory. Two different approaches have been used: one which focuses on the geometric arrangement of atoms in space, and the other which describes the structure in terms of a density function $\rho(r)$ (or more conveniently in terms of the Fourier transform of the density in reciprocal space, $\rho_{\mathrm{q}}$ ) using the Landau theory for crystallization. The former approach was first used by Levine and Steinhardt, ${ }^{[3]}$ who considered the structure to be constructed from a "Penrose tiling" in three dimensions, a 3D analogue of the 2D aggregates of two types of rhombuses, found to have fivefold symmetry, ${ }^{[5]}$ and which produced optical Fourier transform patterns having tenfold symmetry. ${ }^{[6]}$ The quasi-crystal is distinguished by the existence of two characteristic length scales in the structure, whose ratio is an irrational number; for the case of the Penrose tiling, this ratio is the golden mean, $\tau=(\sqrt{5}+1) / 2$. Calculations of the diffraction patterns for such quasi-crystals yield a series of sharp diffraction spots having icosahedral symmetry, in agreement with experiment, even though there is no long-range translational periodicity in the structure. The discovery of fivefold symmetry in diffraction patterns of otherwise apparently perfectly crystalline materials has not just revolutionized the science of crystallography; the theoretical advances required to understand the nature of such quasi-crystalline structures should in turn shed much light on the structure of amorphous materials themselves.

The layman's conception of a "glass" is simply that of an optically transparent, silica-based material; and whilst many amorphous materials produced are indeed of this variety and used in a range of applications, both traditional (window materials, utensils) and advanced technological (glass fibers for optical communications), very many other materials can also be rendered amorphous (see Table 1). ${ }^{[1.7]}$ Some of these non-siliceous amorphous materials have important technical applications. Amorphous selenium films, for instance, form the photoreceptors at the heart of the Xerox process; certain organic glasses are used in an analogous fashion. Some halide glasses have promise as optical fiber materials for use at long wave-
Table 1. Some representative non crystalline solids, their principal bonding type and the temperature $T_{v}$ at which they form when cooled from the vapor, or the glass-transition temperature $T_{\mathrm{g}}$ if cooled from the melt, at an appropriate rate for each material.

\begin{tabular}{llcr}
\hline Material & Type of bond & $T_{、}[\mathrm{~K}]$ & $T_{\mathrm{E}}[\mathrm{K}]$ \\
\hline Tetracene $\left(\mathrm{C}_{18} \mathrm{H}_{12}\right)$ & van der Waals & - & $<180$ \\
Isopentane & van der Waals & - & 65 \\
$\mathrm{H}_{2} \mathrm{O}$ & Hydrogen bridges & - & 140 \\
$\mathrm{Se}$ & covalent & - & 320 \\
$\mathrm{SiO}_{2}$ & covalent/ionic & - & 1450 \\
$\mathrm{BeF}_{2}$ & ionic & - & 600 \\
$\mathrm{Bi}$ & metallic & $<4$ & - \\
$\mathrm{Au}_{0.8} \mathrm{Si}_{0.1} \mathrm{Ge}_{0.1}$ & metallic & - & 300 \\
\hline
\end{tabular}

lengths; amorphous metallic films and ribbons have a variety of prospective applications, including transformer cores and magnetic shielding, superconducting magnetic windings, and high-strength ribbons in fiber-reinforced composites; amorphous films containing a high proportion of chalcogens offer a wide variety of opto-electronic applications, including optical storage media and resists for photo- or electron-beam lithography. Semiconducting amorphous silicon films (containing about 5 percent hydrogen) are currently used as large-area solar cells, for xerography, and as thin-film transistors. In addition many supported catalysts ${ }^{[8]}$ (e.g. ' $\mathrm{V}_{2} \mathrm{O}_{5}$ ' on titania ${ }^{[9]}$ used extensively for selective oxidation of hydrocarbons - cf. Section 8) are unquestionably amorphous in the sense conveyed in this article.

An amorphous solid may be regarded as differing from its crystalline counterpart in containing excess free energy and entropy. These are incorporated during the process of preparation, and this must be sufficiently rapid to preclude the formation of crystalline material by not allowing time for the atoms to reach the lowest energy state, viz. the crystal. Thus, vapor can be rapidly quenched ${ }^{[0,11]}$ to form amorphous thin films using the techniques of evaporation or sputtering, or the glow-discharge or thermal decomposition of unstable molecular species. ${ }^{[1]}$ Crystalline solids can also be rendered amorphous directly by radiation damage by using high-energy neutrons, or electrons or other radiations as in "metamict" minerals such as uranium-containing zircon $\left(\mathrm{ZrSiO}_{4}\right)$. They may be amorphized even by the simple expedient ${ }^{[1]}$ of allowing solvent molecules of crystallization to evaporate at modest temperatures (see below). Solid-state reactions may also produce amorphous solids from starting crystalline materials. An interesting recent development in this area has been the demonstration $^{\text {[121 }}$ that the absorption of hydrogen by a crystalline $\mathrm{Zr}_{3} \mathrm{Rh}$ alloy produces a hydrided amorphous material. The reason for this rather remarkable behavior appears to be that the diffusion coefficients of the metal atoms are less than that of the hydrogen. The most energetically stable crystalline products of the hydriding reaction are the hydride $\mathrm{ZrH}_{2}$ and $\mathrm{Rh}$ metal: in order to form these products, there must be an appreciable degree of metal diffusion, which cannot take place at low temperatures, and so an (X-ray) amorphous product is formed instead. But, perhaps the most common technique for preparing noncrystalline materials is by the rapid quenching of a liquid to form a glass - a method discovered accidentally by the 
Phoenicians to generate glass from a melt of lime, sand, and soda.

\section{The Glass Transition}

Glasses are distinct from other amorphous materials by virtue of a characteristic transition, the so-called glass transition, that they exhibit. ${ }^{[13]}$ At the glass transition temperature, $T_{g}$, the super-cooled liquid departs from the equilibrium behavior (Fig. 2), the actual value of $T_{\mathrm{g}}$ itself depending on the cooling rate. $T_{\mathrm{g}}$ is generally around $0.67 T_{\mathrm{f}}$, where $T_{\mathrm{f}}$ is the fusion temperature. The glass transition also has features apparently similar to those found in second-order phase transitions. For example, $C_{\mathrm{p}}$ and other second derivatives of Gibbs' free energy increase sharply at $T_{\mathrm{g}}$. As seen in Figure 2, the entropy of a liquid would become equal to that of a crystal well above $0 \mathrm{~K}$, provided that there is no intervening transition. This problem is circumvented by postulating the existence of a transition at a temperature $T_{0}$, (thermodynamic transition temperature) which limits the decrease in entropy. In order to avoid the paradoxical situation as to what happens when $T<T_{0}$, in the absence of a transition, one assumes that the glass crystallizes at $T>T_{0}$. What is interesting is that $T_{0}$ values estimated from calorimetry ( $C_{\mathrm{p}}$ vs $\lg T$ plots) are close to those obtained from Equation (1), which describes the temperature variation of viscosity and other dynamic properties:

$\psi=\psi_{0} \exp \left[A /\left(T-T_{0}\right)\right]$

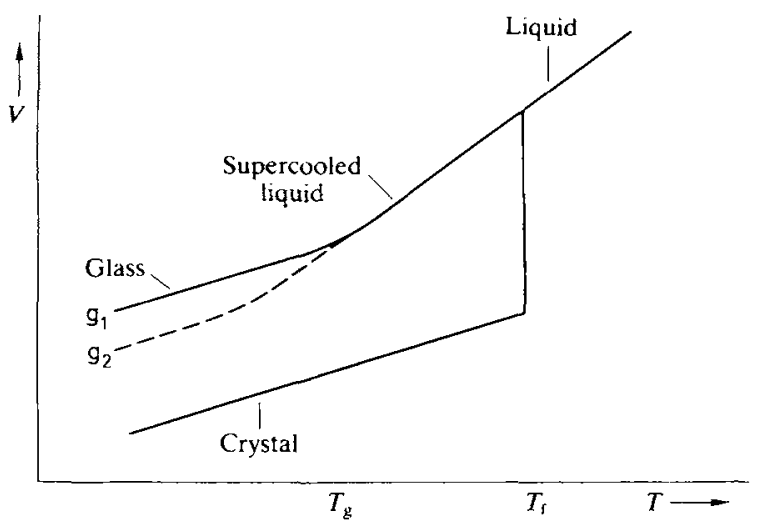

Fig. 2. Schematic illustration of the changes in volume which can occur on cooling a liquid. Crystallization may occur (at $T_{t}$ ), or if the liquid is supercooled below this temperature, glass formation can occur. The temperature marking the break in the slopes of $V$ vs $T$ is termed the glass-transition temperature, $T_{\mathrm{g}}$. Note that the actual value of $T_{\mathrm{g}}$ varies with cooling rate $q$ $\left(q_{1}>q_{2}\right.$; the $T_{\mathrm{g}}$ value in the figure holds for $\left.q_{1}\right)$.

An "ideal" giass has been defined as one in which $T_{\mathrm{g}}$ approaches $T_{0}$. Nonideality of a glass would then be a measure of the entropy frozen in at $T_{\mathrm{g}}$ (this becoming zero when $T_{\mathrm{g}}=T_{0}$ ). The existence of a thermodynamic transition at $T_{0}$ has not been observed so far, since relaxation times are too long below $T_{\mathrm{g}}$. The experimental $T_{\mathrm{g}}$ itself occurs when the relaxation time attains a constant value on the time scale of the experiment.

A variety of relaxation, spectroscopic and other techniques have been employed to investigate the behavior of various properties of glass around $T_{\mathrm{g}}$, but the nature of the glass transition is still not completely understood. ${ }^{[13.14]}$ Noteworthy among the various models proposed for the transition are the free volume model ${ }^{[15]}$ and the configurational entropy model, ${ }^{[16]}$ and these have been reviewed in the literature. ${ }^{[13]}$

The entropy frozen-in below the glass transition has a fairly large non-configurational component. The role of communal entropy has been considered and it is suggested that the communal entropy vanishes at $T_{\mathrm{g}}$, when the liquidlike properties cease to exist. ${ }^{[17]}$ This would require the glass transition to be insensitive to cooling history, but this is contrary to experience. Recent computer experiments ${ }^{[18]}$ as well as electron microscopic studies ${ }^{[19]}$ have indicated the existence of ordered regions or clusters (with noncrystalline motifs) distributed in a tissue material of lower density. When clusters grow in number and size, a congelation to a glass occurs resulting in the elimination of configurational entropy; the tissue material in the intercluster regions could continue to undergo configurational changes, thereby accounting for significant values of the configurational entropy. A cluster model of the glass transition has been developed with the relative size of the cluster as the order parameter. ${ }^{\text {[20] }}$

The term glass transition is generally used in connection with positionally disordered materials, but the transition is also found in solids that are characterized by other degrees of freedom. For example, orientational disorder in plastic crystals may be quenched to yield "glassy crystals" which exhibit glass-like transitions. ${ }^{211}$ Disorder in dipole interactions may be frozen-in to yield dipole glasses (similar to spin glasses), typical examples ${ }^{[22]}$ being $\mathrm{KBr}$ doped with $\mathrm{CN}^{-}$ions or $\mathrm{KTaO}_{3}$ doped with $\mathrm{Li}^{+}$. Frozen liquid crystals are also found to exhibit glass-like transitions. ${ }^{[23]}$ Thus, the glassy state includes long-range disorder of many types while the glass transition manifests itself when relaxational and experimental time scales intersect.

Simulation of the local structures of liquids has its origins in the classic work of Bernal who used mechanical assemblies for this purpose. The importance of non spacefilling symmetries (such as the icosahedral and the pentagonal) to "ordered" aggregates in the amorphous state has been indicated by some of the studies. The role of such aggregates or clusters in understanding the glass transition was pointed out earlier. Computer simulation studies ${ }^{[24]}$ have yielded useful information on the prototype glass transition in simple liquids, although the use of high quenching rates limits the applicability of the results. The high fictive temperatures of the simulated glasses suggest high diffusion rates. Both hard and soft spheres can be compacted into amorphous assemblies, but the characteristic $C_{p}$ discontinuity is absent in these cases. The hard sphere ensemble shows that the diffusivity $D$ does not vary linearly with free volume at low temperatures and $V_{0}^{\text {D }}$ in Equation (2),

$D=A \exp \left[-B /\left(V-V_{0}^{\mathrm{O}}\right)\right]$

seems to represent the Bernal's limiting dense random packing of hard spheres. Computer simulation studies have yet to demonstrate clearly the existence of the $C_{\mathrm{p}}$ 
overshoot, which is the hallmark of the experimental glass transition. Molecular dynamics simulation studies do, however, provide valuable insights into ion movements in glasses. $^{[25]}$

\section{Vitreous Water or Amorphous Solid Water}

Water is by far the most common substance that one can think of and yet its extraordinary properties and behavior continue to confound us. While the various models and predictions have helped in understanding the nature of liquid water, they have always met with failure of one kind or another. Amorphous solid water can be produced by vapor deposition of dilute gas molecules on a cold substrate. ${ }^{[26]}$ The resulting material appears glassy and is amorphous to X-rays. A glass transition has been noticed in such deposits ${ }^{[27]}$ and the $T_{\mathrm{g}}$ predicted by the extrapolation of data for binary salt solutions $(139 \mathrm{~K})$ was until recently considered to be consistent with the $T_{\mathrm{g}}$ obtained by thermal measurements. ${ }^{[28]}$ The heat capacity of amorphous solid water measured by adiabatic calorimetry does indeed show an increase, as expected of a glass transition. ${ }^{[29]}$ The extrapolated $T_{\mathrm{g}}$ was however not considered to be compatible with the heat capacity of liquid water. ${ }^{[28]}$

The structure of amorphous solid water prepared by vapor deposition is not different from what is expected for the low-temperature ground state for liquid water. Ultrafast quenching of samples of liquid water has been found to produce amorphous material similar to that prepared by vapor deposition; furthermore, crystallization does not occur during such cooling. ${ }^{[30.31]}$ Crystallization of amorphous solid water occurs around $162 \mathrm{~K}$. What is rather puzzling about amorphous solid water is the very recent observation $^{[32]}$ that no thermal manifestation of the glass transition occurs anywhere in the temperature region predicted by the extrapolation of the data on binary salt solutions. ${ }^{[32]}$ It appears that water in the amorphous state is not any easier to understand than in the liquid state.

\section{The Structure of Amorphous Materials}

The determination of the structure of amorphous materials has remained a challenge ever since $X$-ray diffraction was first used as a structural tool for such solids in the 1930's. The problem lies in the fact that in the absence of periodicity (i.e. regularly spaced planes of atoms), the scattering of $\mathrm{X}$-rays (or neutrons or electrons) produces diffuse haloes of scattered intensity, rather than the sharp Bragg spots or lines characteristic of single crystals (see Fig. 3 and 4). Expressed in terms of the scattering vector, $k=4 \pi \sin \theta / \lambda$, in reciprocal space, the intensity is a damped series of broad oscillations superimposed on the square of the atomic form factor, $f_{\mathrm{x}}^{2}(k)$. Real-space information can be obtained from the scattered intensity data by Fourier transformation after suitable normalizations have been carried out. The resulting curve, the radial distribution function (RDF), $J(r)$, exhibits peaks (Fig. 3b) at distances corresponding to the average interatomic separation for the first shell, second shell, etc., superimposed on a background curve, the average density parabola, $4 \pi r^{2} \rho^{0}$. Although there is a severe problem for higher-lying peaks in that different shells can contribute to the same peak in the RDF, nevertheless, for the first (and second) peak, the area under the peak in the RDF gives the coordination number directly.

The difficulty with a structural interpretation of the RDF of an amorphous material lies in the fact that the RDF is an average quantity; it describes the spatially averaged structural arrangement about a given origin atom, and is a chemical average over all types of atom in the sample. Just as in endeavoring to describe the structure of a liquid via RDF's, we proceed likewise with amorphous solids. The very same obstacles encountered in specifying the structure of liquids are also encountered here: the RDF itself is not sufficiently sensitive to variations in the model, partly because it is a spatially-averaged property.

Since very many amorphous materials contain several
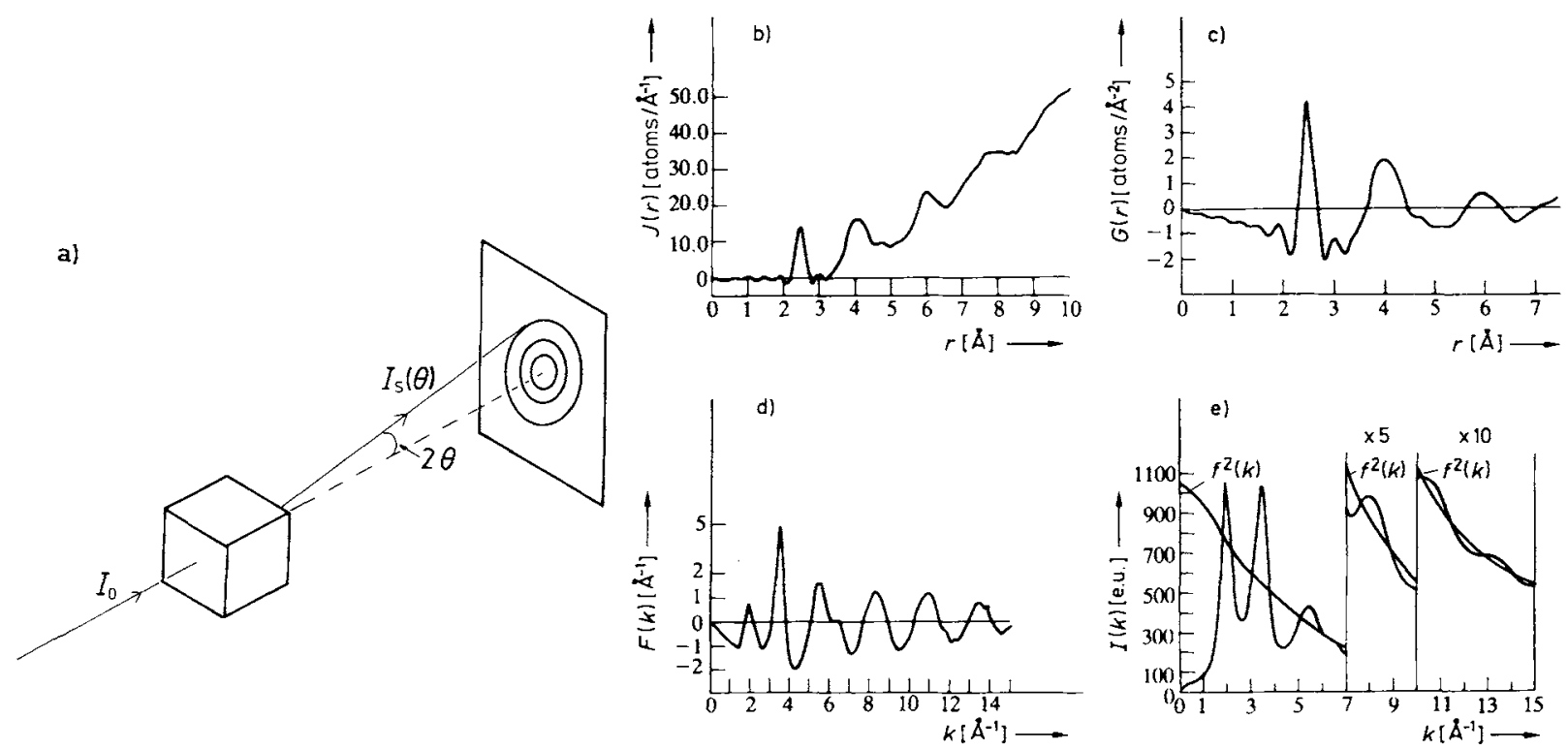

Fig. 3. (a) Schematic illustration of the experimental arrangement used in a diffraction measurement: (b)-(e) $X$-ray scattering data for a- $(j e$ (after Temkin et al. [33]). (b) the RDF (cf. Fig. Ic); (c) the reduced RDF; (d) the reduced scattering intensity; (e) the total (corrected) measured scattering intensity. 
a)

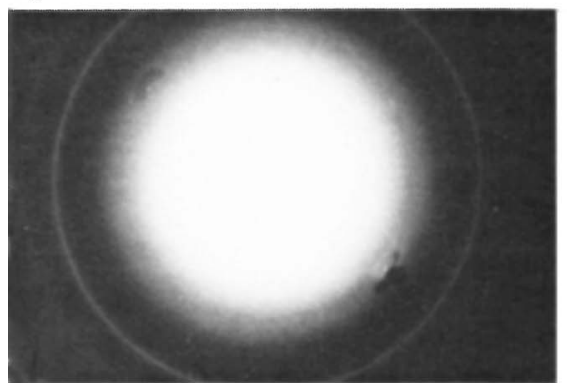

b)

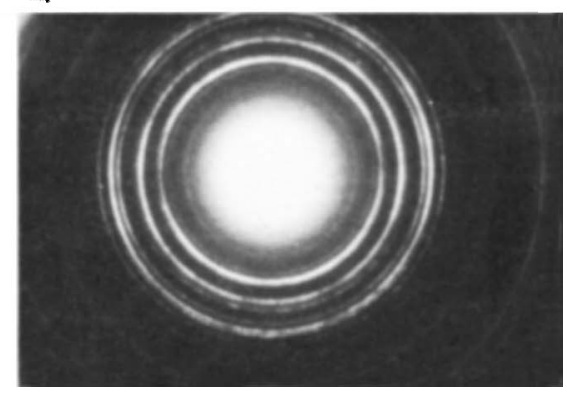

Fig. 4. Experimental electron diffraction patterns of vapor deposited tetracene films, measured at (a) $20 \mathrm{~K}$ and (b) $240 \mathrm{~K}$. Note that the film formed at the lower temperature is amorphous while the film produced at the higher temperature exhibits clear signs of polycrystalinity. The growth of crystalline films at higher temperatures is more clearly seen in (c), where the scattering intensity is shown as a function of scattering angle $s=(\sin \theta) / \lambda$ for films deposited at the various temperatures indicated [10].

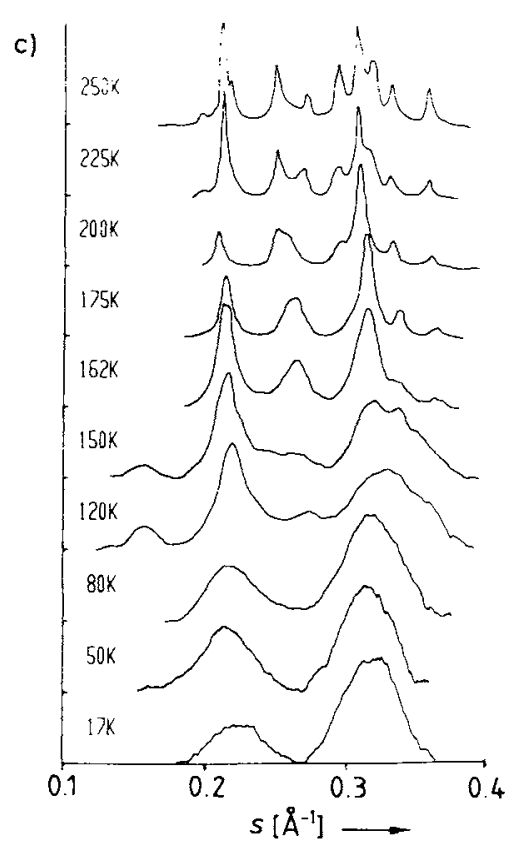

components, this lack of chemical specificity of conventional diffraction techniques is a severe handicap. One technique which is chemically specific and of considerable promise is the method of extended X-ray absorption fine structure (EXAFS). ${ }^{[34-36]}$ In this, X-rays from a broad-band source (e.g. synchrotron radiation or Bremsstrahlung from a conventional $\mathrm{X}$-ray generator) eject photoelectrons from an atom if the $X$-ray photon energy is greater than the binding energy of a core electron (say from the K-shell). The photoelectron will be backscattered from any surrounding atoms and interference can occur between outgoing and backscattered states, depending on the energy of the initial X-ray (and hence the kinetic energy of the photoelectron); in this way the $\mathrm{X}$-ray absorption coefficient is modulated, leading to "fine structure" on the high energy side of the (K) edge (see Fig. 5). The method is chemically specific because the X-ray absorption edges of different atoms fall at different energies, and hence the EXAFS for different elements can be well separated in energy (Fig. $5 b$ ). Real-space structural information can be obtained from the experimental EXAFS data by Fourier transformation after suitable background subtractions and normalizations. The method is particularly sensitive to the nearest-neighbor environment, but does not probe interatomic correlations beyond about the third nearest neighbor. It is a technique particularly suited for the structural study of disordered materials since it does not depend for success upon the presence of long-range order.

Another technique which is capable of elucidating the structural characteristics of simple (elemental or binary) amorphous materials is electron Compton scattering. ${ }^{[38,39]}$ Compton scattering generally, whether it involves scattering of $\gamma$ (or X-ray) photons or high energy electrons, can yield $^{139]}$ the ground state wave-functions of the material under investigation. Even when there is no long-range order, the local electronic environment surrounding a central atom can be probed via the so-called reciprocal form fac- tor. As the form factors for the corresponding crystalline materials are well known by separate determinations, the experimentally derived Compton profile yields the reciprocal form factor, $B(z)$, as defined in Equation (3).

$B(z)=\int_{-\infty}^{+\infty} \exp (i p,-z) J\left(p_{z}\right) \mathrm{d} p_{z}=\iint_{-\infty}^{+\infty} \psi(r-z) \psi(r) \mathrm{d} r$
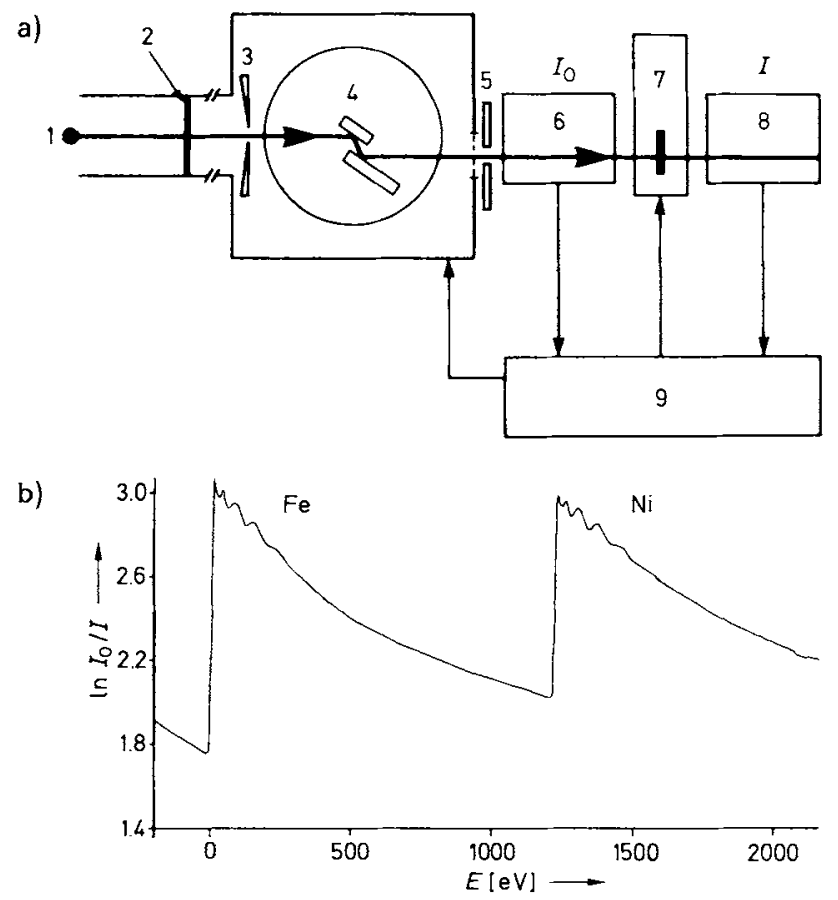

Fig. 5. (a) Schematic illustration of the experimental arrangement employed for transmission EXAFS measurements. 1: Source point of synchroton radiation; 2: Be window; 3: slit; 4: monochromator channel-cut crystal; 5: mask; 6, 8: ionization chambers 1 and 2 in which intensities of the incident and transmitted X-rays, respectively, are measured; 7 : sample; 9 : computer. (b) $\mathrm{K}$-edge EXAFS spectra of $\mathrm{Fe}$ and $\mathrm{Ni}$ in $\mathrm{Fe}_{40} \mathrm{Ni}_{40} \mathrm{~B}_{20}$ glass at $77 \mathrm{~K}$ (after [37]). $E$ signifies energy above $\mathrm{Fe} \mathrm{K}$ edge. 
In other words, the Compton profile $J\left(p_{z}\right)$ [Equation (4)], defined as the projection of the momentum density $\rho(p)$ onto the scattering vector $p_{2}$, where

$J\left(p_{,}\right)=\prod_{-\infty}^{+\infty} p(p) \mathrm{d} p_{\mathrm{x}} \cdot \mathrm{d} p_{y}$

can be determined directly from experimental Compton profiles. The reciprocal form factor is ${ }^{[38]}$ the overlap of the ground-state wave-function with itself as a function of the distance $r$. For the amorphous material, this gives us a means of ascertaining the local bonding. In this way it was recently established ${ }^{[40]}$ that noncrystalline elemental carbon involved predominantly $\mathrm{sp}^{2}$ not $\mathrm{sp}^{3}$ bonding (i.e. was essentially graphitic).

Another chemically specific structural probe which has great potential for the study of amorphous solids is nuclear magnetic resonance (NMR), ${ }^{[4]]}$ which is capable of giving high-resolution (liquid-like) spectra if special ancillary techniques such as magic-angle spinning or multiple-pulse sequencing are employed with modern pulse (Fourier transform) spectrometers ; ${ }^{1421}$ the method is again sensitive mainly to the nearest-neighbor environment. Earlier broad-line NMR studies ${ }^{[4]}$ yielded important, but somewhat limited, structural information, particularly concerning borate glasses. ${ }^{29} \mathrm{Si}$ magic-angle-spinning NMR (MASNMR) is proving to be particularly useful in both crystalline and noncrystalline siliceous solids. ${ }^{[44]}$ There is a wellestablished correlation ${ }^{[45]}$ between the anisotropic ${ }^{29} \mathrm{Si}$ chemical shift ( $\delta$ values; standard: tetramethylsilane) and the $\mathrm{T}-\mathrm{O}-\mathrm{T}$ angle $(\mathrm{T} \equiv \mathrm{Si}$ or $\mathrm{Al})$ given by

$\delta=-25.44-0.5793(\theta / \mathrm{deg})$

so that quantitative deductions about local structure can be made from the observed ${ }^{29} \mathrm{Si}$ chemical shifts. When zeolite $\mathrm{A}$, an aluminosilicate (having the empirical formula $\mathrm{Na}_{12} \mathrm{Al}_{12} \mathrm{O}_{48} \cdot 27 \mathrm{H}_{2} \mathrm{O}$ ) crystallizes out from an amorphous gel precursor the change in $\mathrm{T}-\mathrm{O}-\mathrm{T}$ bond angles in going from $\mathrm{Si}(\mathrm{O}-\mathrm{Al})_{4}$ (gel) to $\mathrm{Si}(\mathrm{O}-\mathrm{Al})_{4}$ (zeolite $\left.\mathrm{A}\right)$ results in a readily detectable change (from -85.1 to $-90.8 \mathrm{ppm}$ with respect to tetramethylsilane TMS) in the ${ }^{29} \mathrm{Si}$ isotropic shift characteristic of the $\mathrm{Si}(\mathrm{OAl})_{4}$ environment. Conversely, when zeolite- $\mathrm{A}$ is rendered amorphous by high-temperature (hydrothermal) treatment typical of that employed in procedures for burying radioactive waste, the chemical shifts (and line widths) change as shown in Figure 6. a)

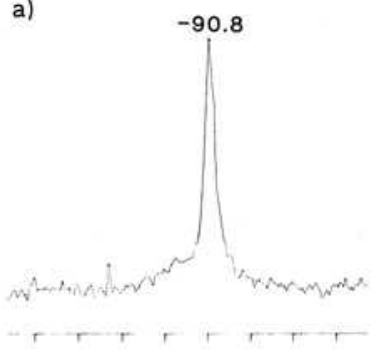

b)

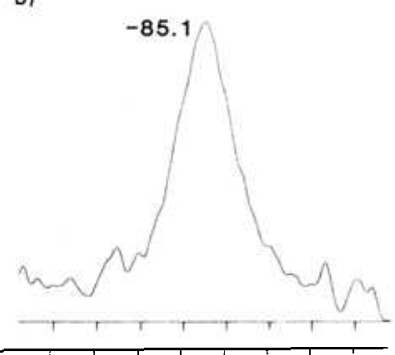

Fig. 6. Section of the "'Si MAS-NMR spectrum of zeolite A (a) before and (b) after amorphization (e-g., as in the sealing of radioactive waste for disposal) [8]. $\delta$ values, standard TMS.
Dupree and Pettifer ${ }^{[46]}$ have recently shown how line shapes in ${ }^{29} \mathrm{Si}$-MAS-NMR spectra can be used to set limits to the $\mathrm{T}-\mathrm{O}-\mathrm{T}$ angles present within a glass.

MAS-NMR is ideally suited to probe local site environments and to determine changes in site occupancies during solid-state transformations. So far, most work has been done on systems containing ${ }^{27} \mathrm{Al}$, which, being a quadrupolar nucleus, relaxes rapidly, thereby yielding good-quality spectra in relatively short-duration experiments. ${ }^{[44]}$ But there is clearly abundant scope for the exploration of organic glasses (via the ${ }^{13} \mathrm{C},{ }^{1} \mathrm{H},{ }^{2} \mathrm{H}$ and ${ }^{3} \mathrm{H}$ nuclei) and for a variety of inorganic systems with the sixty or so nuclei that exhibit magnetic resonance of acceptable intensity.

\section{Models of the Amorphous State}

One of the first models to describe the amorphous state was proposed by Zachariasen in 1932 for the case of covalently bonded solids. ${ }^{[47]}$ The structure was supposed to be formed from polyhedra (e.g. $\mathrm{SiO}_{4}$ tetrahedra in the case of $\mathrm{SiO}_{2}$ ), connected together to form a "continuous random network" (CRN), in which long-range order is destroyed by allowing freedom of rotation of neighboring polyhedra about the connecting atom, i.e. the T-O-T angle is allowed to take up a variety of values. Note that the term "random network" is somewhat of a misnomer here, for the structure is not truly random in the statistical sense; well-defined local order exists in the glass by virtue of the existence of the polyhedra, and if the polyhedra connect together to form say rings, then the distribution of torsion ("dihedral") angles for neighboring units is not uniform (i.e. random), but instead certain dihedral angles are preferred.

In 1971 , Polk ${ }^{[48]}$ demonstrated that it was possible to build an extended continuous random network (consisting of 440 atoms), based on an invariant coordination number of four, for tetrahedrally bonded amorphous semiconductors such as silicon and germanium. The key point about Polk's work was that even when all bond lengths were kept close to their equilibrium value (for the crystalline state) no intolerable bond-length strain was produced, provided a modest spread in angles was permitted. Significantly, five-, six-, and seven-membered rings were present in such a continuous network - there are only six-membered rings in the crystalline semiconductors but, even more important, the density of the resulting noncrystalline structure differed from that of the corresponding (diamond-like) structure by about one percent. The message here is that, for covalently bonded, relatively simple amorphous solids ( $\mathrm{Si}, \mathrm{Ge}, \mathrm{SiO}_{2}, \mathrm{GeO}_{2}$, etc.) the short-range order is very similar to that which prevails in the corresponding crystals. (Even in the ionic noncrystalline solid formed by $\mathrm{BeF}_{2}$, $4: 2$ coordination is still retained in view of the magnitude of the respective ionic radii, $r_{\mathrm{Be}^{2+}}=0.27 \AA, r_{\mathrm{F}^{-}}=1.31 \AA$, thereby favoring 'tetrahedral' coordination of fluoride ions around the central cations.)

The question of "intermediate-range" order, beyond the short-range local structure characterized by the polyhedral units in covalent glasses, is also of much current interest. In particular, there is a growing body of evidence for the 
existence of rather well-defined clusters of atoms, containing 10 or more atoms, in the structure of certain covalent glasses (e.g. Ge-Se alloys); vibrational spectroscopy as well as diffraction experiments indicate their presence, and, as we have seen, certain aspects of glass-transition be havior can be understood in terms of the existence of clusters (see Section 2).

Polymeric solids, typified by polystyrene, poly(vinyl chloride) and poly(vinylidene chloride) more often than not are noncrystalline-some chemical ingenuity is required to produce crystalline polymers-and here the "random coil model" is the most appropriate description (see Fig. 7). Like the continuous random network model, the random coil model implies a homogeneous, singlephase solid. It is superior to an earlier, contending theory proposed to account for the behavior of polymeric organic glasses, namely the microcrystalline model, in which microcrystallites of crystalline polymer are embedded in a surrounding noncrystalline matrix. This is incompatible with all the experimental facts for organic glasses, as it is also for most other kinds of glasses. There are, however, partially crystalline polymers (e.g. poly(4-methyl-1-pentene) and poly(ethylene glycol)) in which well-defined coiled regions are embedded within the randomly coiled analogue. Likewise, in polyethylene it is well-known that there are amorphous regions connecting the ordered, folded-chain elements in the so-called crystalline material.

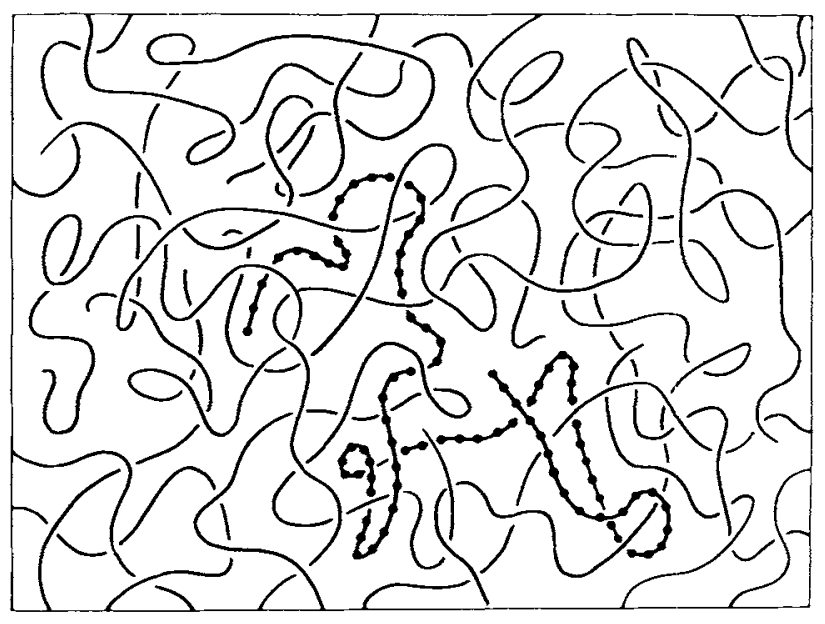

Fig. 7. Schematic diagram of the random-coil model for the structure of polymeric glasses. One chain is clearly marked for ease of visualization (after [7])

Ionic-covalent, noncrystalline solids are typified by amorphous zeolites (general formula $\mathrm{M}_{\mathrm{x} / \mathrm{n}}\left(\mathrm{AlO}_{2}\right)_{\mathrm{x}}\left(\mathrm{SiO}_{2}\right)_{\mathrm{y}} \cdot \mathrm{m} \mathrm{H}_{2} \mathrm{O}$ ). Here, there are several levels of structural order in the parent crystalline form: local order, such as tetrahedrally coordinated $\mathrm{Si}^{4+}$ and $\mathrm{Al}^{3+}$ ions forming $\mathrm{TO}_{4}$ tetrahedra, and variously connected tetrahedra which produce a range of possible polyhedral units (Fig. 8a) that can, in turn, be joined to form a three-dimensional solid (Fig. 8b). In partially amorphized zeolitic structures the scale of residual local ordering is such as to be quite amenable to highresolution electron microscopy (Fig. 9a). Islands of crystalline structure, as well as more or less isolated patches consisting of a few (or a single) polyhedral units, can be di-

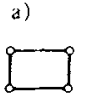

S4R

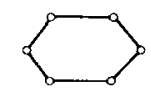

S6R

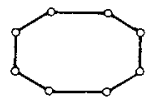

S8R

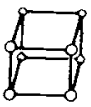

D4R

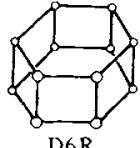

D6R b)
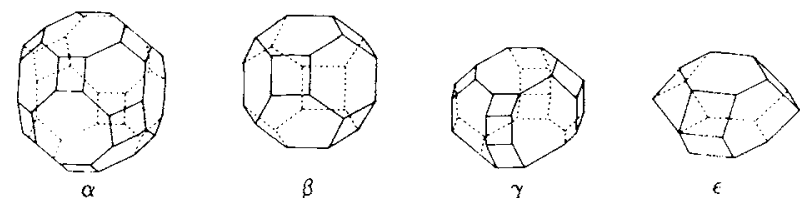

Fig. 8. (a) Simple building blocks and (b) various kinds of polyhedra formed when $\mathrm{TO}_{4}$ tetrahedra are corner-linked. S, D and $\mathrm{R}$ denote single, double and ring respectively. Thus a hexagonal prism, being a double six ring, is designated D6R. The $\beta$ cage is made up of eight $S 6 R$ and six $S 4 R$.

rectly identified ${ }^{[49-51]}$ in such micrographs. Moreover, if strongly scattering ions (e.g. $\mathrm{UO}_{2}^{2+}$ ) are introduced, by ion exchange, into the microcrystalline region of a zeolitic glass, the locally ordered parts of the solid are readily seen $^{\left[{ }^{1]}\right.}$ (Fig. 9b). For comparison Figure 9c shows an HREM image of dehydrated silica gel which is thought to be a bona fide example of the continuous random network model. ${ }^{149]}$

Glassy metals and metallic alloys are best thought of, structurally, as being made up of a dense random packing of hard spheres. The notion of dense random packing (DRP) figured eminently in the classic work of Bernal ${ }^{[52]}$ on the structure of monoatomic liquids. Bermal's work on liquids involved ball bearings packed into rubber bladders, kneaded, set in black paint with the positions analyzed by hand and eye. Later variants of this approach entailed computer simulations. All these approaches showed that it is possible to generate a randomly-packed model which is sufficiently dense (64 per cent occupancy of available space compared with 74 per cent in cubic or hexagonal close packing of spheres) to reproduce experiment. Such a DRP model involves no crystalline regions provided the spheres are not bounded by a regular surface. (Spheres poured into a cylinder can be densely, randomly packed: this is not the case if poured into a cubic or rectangular box!)

Whereas it is well known that, in the closest packing of spheres (as in crystalline metals such as $\mathrm{Ag}, \mathrm{Au}$ and $\mathrm{Pt}$ ), there are 'holes' which are tetrahedral and octahedral in shape (as indeed there are in close-packed anions, e.g., in the structures of $\mathrm{Fe}_{2} \mathrm{O}_{3}$ and $\mathrm{Fe}_{3} \mathrm{O}_{4}$ ), Bernal observed that, in the DRP model, holes were of five distinct types. In addition to the tetrahedral and octahedral holes (see Fig. 10) there are three others. All these are polyhedra with equal triangular facets (i.e. deltahedral objects) which are small enough not to admit another sphere of the same size. These are known as Bernal's 'canonical holes'. It transpires that an almost indefinite number of structures, each with almost identical nearest-neighbor distances, can be formed by packing the five deltahedra together. Finney constructed a dense randomly packed structure, composed of 7994 'atoms' with a packing efficiency of 44 percent. The (total) radial distribution function (see Fig. 11a) for this structure compares well with that determined experimentally for amorphous $\mathrm{Ni}_{76} \mathrm{P}_{24}$, although the agreement 


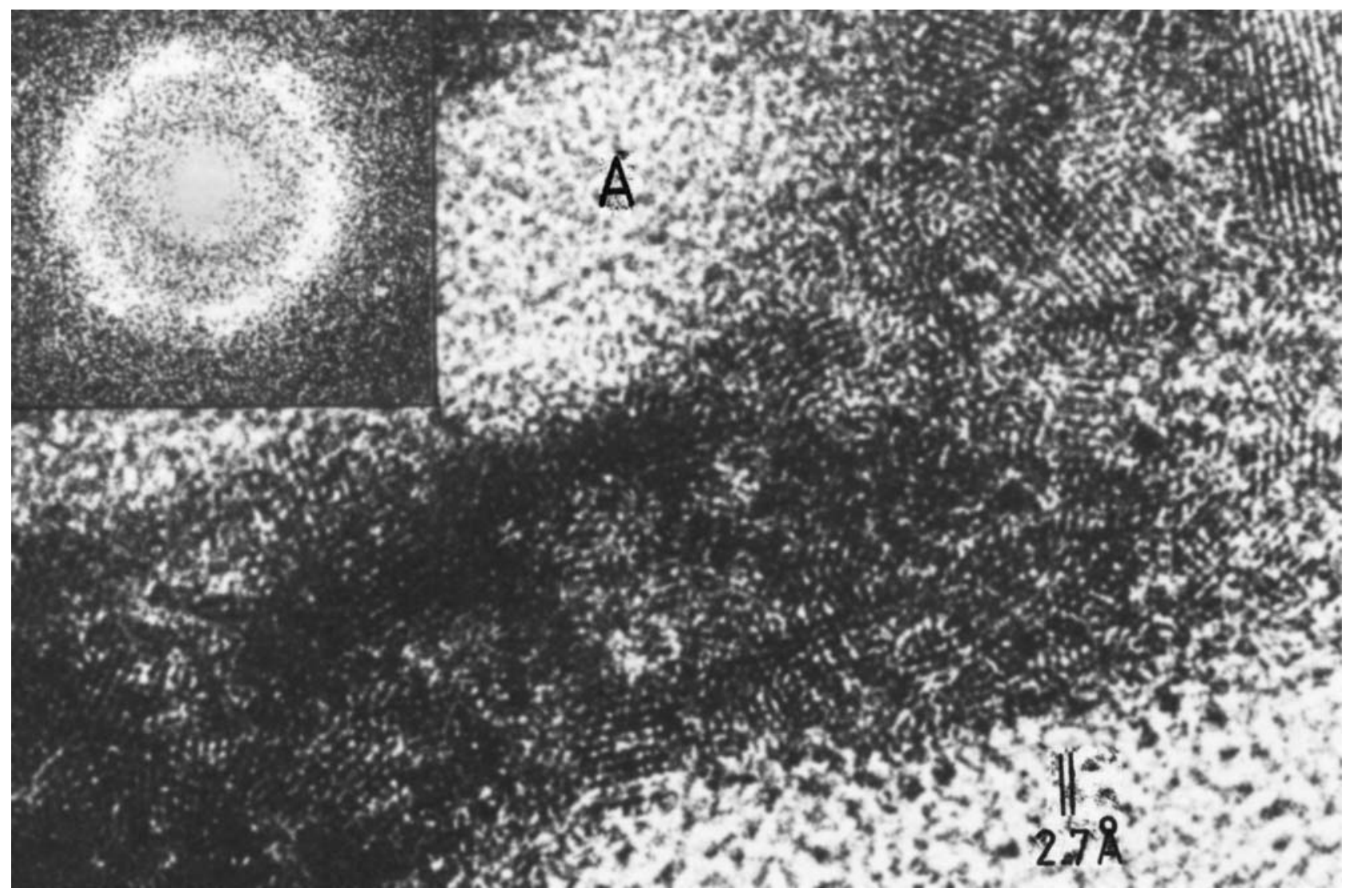

Fig. 9a. High resolution electron micrograph (HREM) of amorphous zeolite A

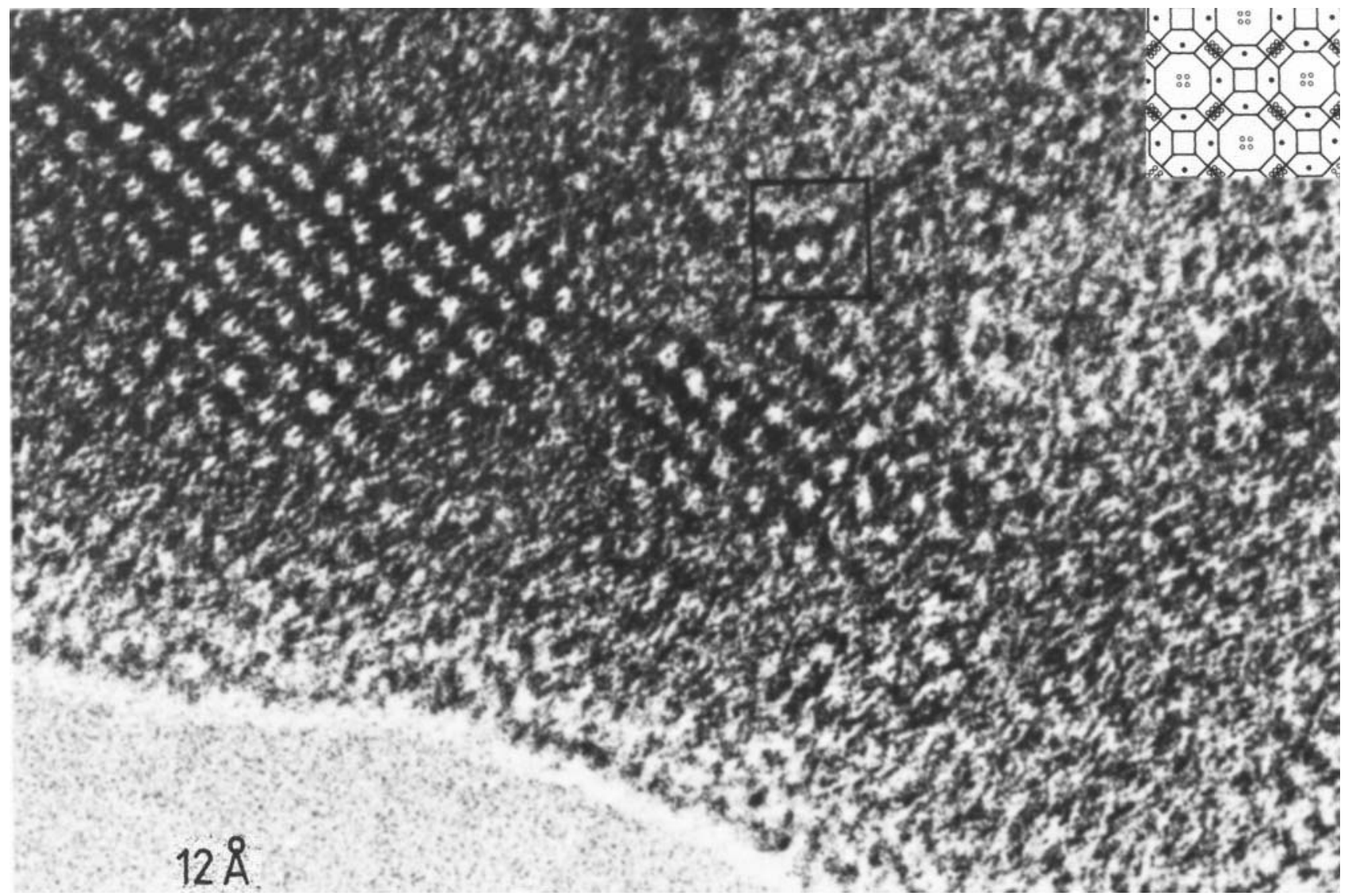

Fig. 9b. High resolution electron micrograph of microcrystalline zeolite Y. A "supercage" is framed. 


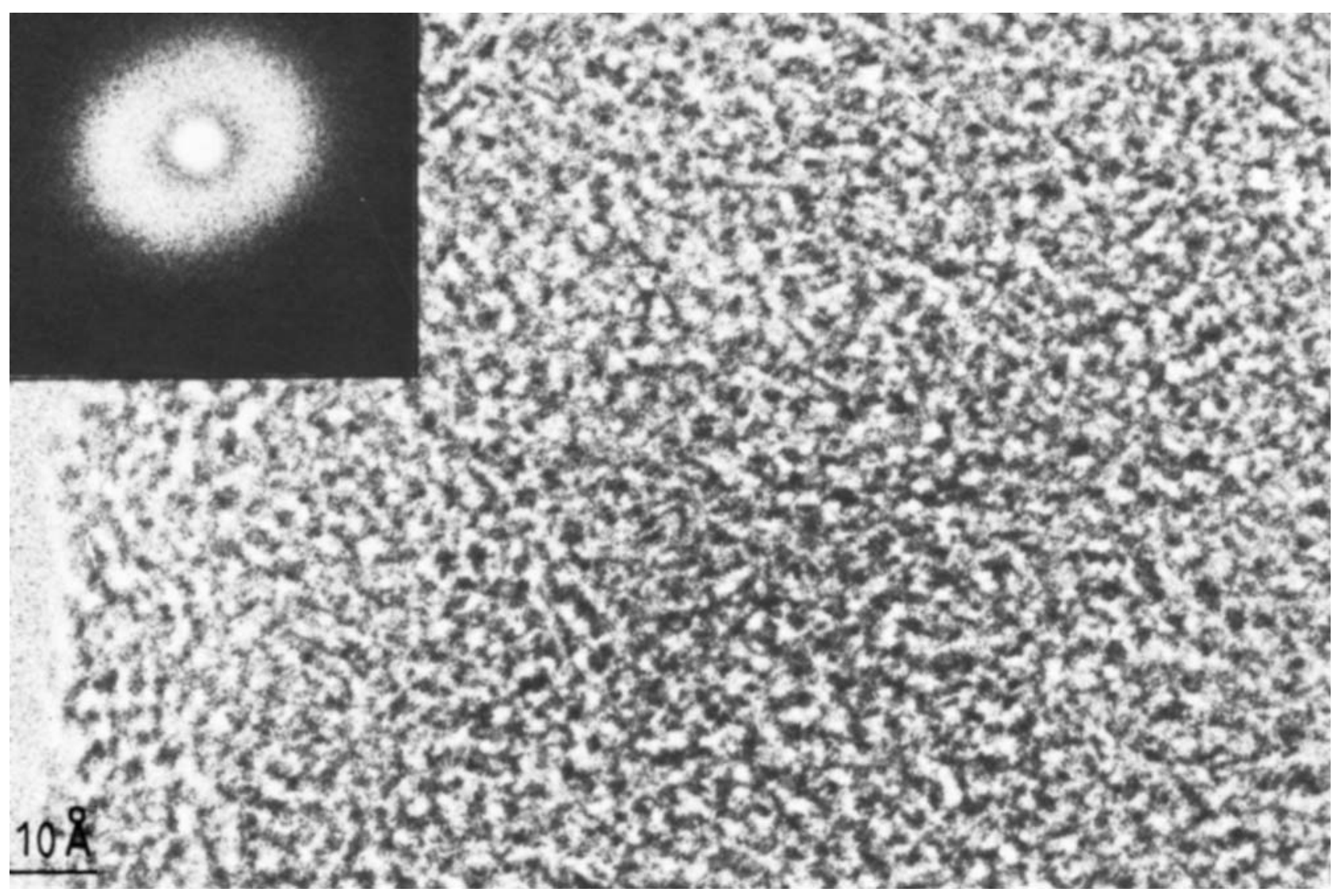

Fig. 9c. High resolution electron micrograph of random network amorphous region of dehydrated silica gel.

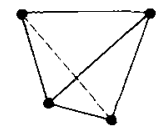

a)

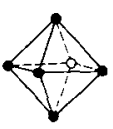

b)

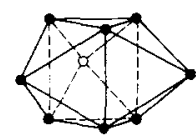

c)

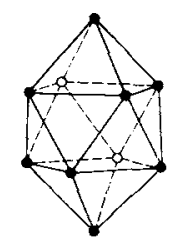

d)

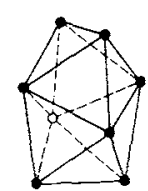

e)
Fig. 10. The five "canonical" holes described by Bernal. They are a) tetrahe dron; b) octahedron; c) trigonal prism (capped with three half-octahedra); d) Archimedian anti-prism (capped with two half-octahedra); e) tetragonal dodecahedron. for the individual partial pair correlation functions is less good (Fig. 11b and c).

The DRP model does, however, certainly seem to account well for the structure of amorphous metal-metal alloys, say $\mathrm{Zr}-\mathrm{Cu}$. However, there is an important class of amorphous metallic alloys which do not appear to be described by this model--these are the so-called "metglas" alloys formed from approximately $80 \%$ transition metal and $20 \%$ semimetal (e.g. B, Si, P), this being approximately the eutectic composition. In these materials, chemical or-
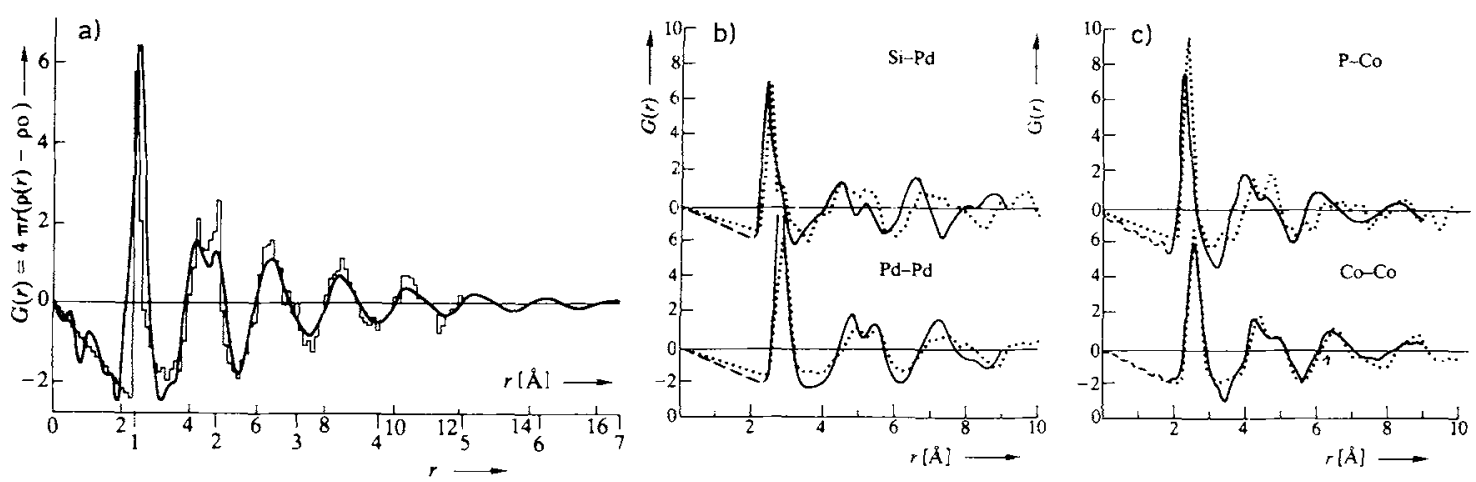

Fig. 11. Reduced radial distribution curves $G(r)$ (cf. Fig. 3c). (a) Calculated for the Finney DRP model compared with the experimental curve for a Ni-P glass; $(r$ is given in units of the diameter of the sphere); (b) and (c) reduced RDFs calculated for the Gaskell trigonal prismatic model compared with experimental curves for $\mathrm{Pd}-\mathrm{Si}$ and Co-.P glasses. 
dering beyond that expected for the DRP model appears to exist, and a detailed understanding of the structure is lacking, although it has been suggested that crystalline motifs such as trigonal prismatic units might exist in the glass. It is well known ${ }^{[53]}$ that the structure of crystalline transition metal-semimetal alloys, such as cementite $\left(\mathrm{Fe}_{3} \mathrm{C}\right)$ or $\mathrm{Fe}_{3} \mathrm{P}$ (not quite the same composition, note, as that of the glasses, which is approximately $\mathrm{Fe}_{4} \mathrm{P}$, for example) comprise trigonal prismatic structural units consisting of $\mathrm{MT}_{6}$ arrangements, in which $\mathrm{M}$ is the semimetal and $\mathrm{T}$ the transition metal (see Fig. 10c). It has been suggested by Gas$k^{2} l^{[53]}$ that the structure of the glassy alloys also consists primarily of such well-ordered local units (see Fig. 11b). In this way, semimetal-semimetal nearest-neighbor avoidance is achieved (consistent with diffraction evidence), which is not the case for the DRP model, in which it is supposed that the small semimetal atoms, such as $P$, reside in the canonical polyhedral holes in the structure, and as such, there is nothing to stop them being nearest neighbors. It is of interest to note in passing that the trigonal prismatic model for the structure of metallic glasses can be thought of as a bridge between the CRN and DRP approaches; it can be regarded as a random network of trigonal prismatic structural polyhedra (somewhat distorted to allow complete connectivity), or alternatively, since the trigonal prism is one of the Bernal canonical holes, it can be regarded as a special case of a DRP, in which only one type of polyhedron is allowed to exist. The precise structure of metal glasses is, however, still the subject of some dispute.

The general question of the extent of chemical ordering in amorphous materials is of considerable interest. In crystalline solids, nonstoichiometry can be achieved by several distinct means. ${ }^{[54]}$ Certain crystalline solids can be progressively denuded of one of their constituents leaving the structure more open but in broad architectural terms quite intact. Some perovskites $\left(\mathrm{ABO}_{3}\right)$, e.g. $\mathrm{CaMnO}_{3}$, behave ${ }^{[55,56]}$ in this fashion when reduced by $\mathrm{H}_{2}$ or hydrocarbons to yield $\mathrm{ABO}_{3-\mathrm{x}}(0<\mathrm{x}<0.5)$, the residual oxygen vacancy sites adopting ordered structures. Another way in which gross nonstoichiometry can be accommodated is for local, but well-defined collapsed regions, such as crystallographic shear planes, to be introduced at intervals through the bulk solid whilst, at the same time, preserving the local coordination, e.g. $\mathrm{MO}_{3} \rightarrow \mathrm{MO}_{3 \mathrm{n}-1}, \mathrm{MO}_{3 \mathrm{n}-2}, \mathrm{MO}_{3 \mathrm{n}-3}$, etc. $(\mathrm{n}=3 \ldots 20)$. In amorphous materials however, gross nonstoichiometry, which can be more or less continuously varied, can be achieved in a different manner, there being no translationally repeated sharply-defined architectural unit in the structure to begin with. This continuous variation can be realized, for the case of a covalent solid, by the local environment changing, so that, say, more like-atom bonds of the elements in excess of that corresponding to stoichiometry are produced.

\section{Structural and Chemical Changes in Noncrystalline Solids}

It should not be thought that the structure of an amorphous solid is immutable. For instance, a glass quenched rapidiy from the melt is in a metastable state, in several senses. If it is held at a temperature near $T_{\mathrm{g}}$ for a sufficient length of time, the structure "stabilizes", and the glass evolves to a state characteristic of a material produced using a slower quench rate. Apart from being able to explore such local, shallow potential minima, the glass is of course energetically metastable with respect to the crystalline state, and will tend to crystallize if held at or above $T_{\mathrm{g}}$ for a sufficiently long period of time. An as-deposited amorphous thin film can also have its structure altered in this manner by thermal means.

There are indications ${ }^{[57]}$ that considerable motion, including transport and agglomeration of ions may occur rather freely within noncrystalline structures. Some polyethylene oxide and organic polycarbonate glasses, for example, permit facile migration of ions such as $\mathrm{Li}^{+}$at low temperatures (ca. $60-80^{\circ} \mathrm{C}$ ) and this fact is harnessed ${ }^{[581}$ in certain types of solid-state batteries (e.g. those utilizing metallic lithium and $\mathrm{TiS}_{2}$ into which $\mathrm{Li}^{+}$species are readily incorporated).

Another phenomenon, worthy of further investigation, is the precipitation from glassy materials at relatively very low temperatures, of phases which are normally prepared at much higher temperatures. Thus, FeS crystallizes ${ }^{[59]}$ out of a Fe-containing $\mathrm{As}_{2} \mathrm{~S}_{3}$ noncrystalline solvent. Certain glasses, based on alloys containing $S$ or Se, suffer ${ }^{[60]}$ structural changes when irradiated by light. The nature of such photostructural changes, which can be removed by annealing at $T_{\mathrm{z} z}$ is, at present, uncertain, although the photoinduced changes are not confined to the structure alone since changes in the optical properties and even in chemical properties (e.g. photo-enhanced dissolution of metals) are also observed. These effects have many potential technological applications, including optical storage media and resists for photo- or electron-beam lithography.

\section{Electronic Properties}

The effect of structural disorder on the electronic properties of solids may at first sight be thought to be small: glasses can be variously metals, semiconductors or insulators, like crystals, and indeed even the overall shape of the bands of electron states in an amorphous solid (e.g. Si) can be broadly similar to that observed in the corresponding crystal (see Fig. 12). However, the presence of disorder does have ${ }^{[6[-63]}$ at least two very important ramifications: electrons can be spatially localized by the existence of spatial (topological) disorder, and the absence of periodicity means that the reciprocal lattice vector, $k$, is no longer a good quantum number. (It should be noted in passing that disorder can produce exactly the same effects for vibrational excitations, i.e. phonons.)

There obviously cannot exist a reciprocal lattice if a periodic spatial lattice is absent, as is the case for a disordered material, and thus the reciprocal lattice vector $k$ becomes redundant as a description of the solid and of the excitations (electronic or vibrational) which occur within it. The unimportance of $k$ for disordered solids leads to an immediate paradox, namely, why theoretically should a forbidden energy gap appear between bands of states for an 
amorphous material? It is common experience that this is the case: for instance, common silicate glass is transparent to photons in the visible spectrum (and indeed has a band gap of almost $10 \mathrm{eV}$ ). The paradox arises because electrons in crystalline solids are conventionally described within the Bloch formalism, i.e. the electron wave function has the form of a plane wave, modulated according to Equation (5) by the function $\mu_{\mathrm{k}}(r)$, which has the periodicity of the underlying lattice:

$\psi_{\mathrm{k}}(r)=\mu_{\mathrm{k}}(r) \exp (\mathrm{i} k \cdot r)$

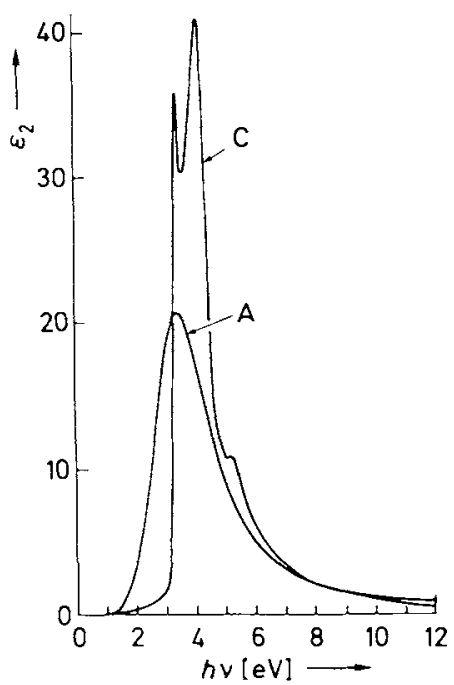

Fig. 12. Optical absorption spectra for crystalline (C) and amorphous (A) silicon. The shape of the absorption bands is related to the joint density of states. Note the sharp features in the spectrum of the crystal (a result of the constraint of $k$-conservation and the existence of singularities in the joint density of states) are absent in the spectrum of the amorphous material as a result of the loss of $k$-conservation ( $\varepsilon_{2}$ is the imaginary part of the dielectric constant).

Interference between Bloch waves results in standing waves being set up and an energy difference (band gap) arises for the cases where the electron density is concentrated near the ion cores and where it is concentrated between the cores. Such an explanation is manifestly inapplicable for noncrystalline materials, and instead of invoking long-range order and its effect on the electrons, the answer must be sought in the local order in a glass. We have already seen that indeed the local order of (covalent) materials is very similar for both the amorphous and crystalline forms; for example $\mathrm{Ge}$ and $\mathrm{Si}$ in both forms consist of atoms in tetrahedral coordination. If just two (shortrange) interactions are considered between the $\mathrm{sp}^{3}$-hybrid orbitals emanating from each atom, one an intersite interaction $\left(V_{2}\right)$ responsible for the separation of bonding and antibonding orbitals and the other $\left(V_{1}\right)$ an intrasite interaction responsible for the width of the bands (see Fig. 13), then according to Weaire and Thorpe a gap between valence and conduction band is predicted as long as the following condition is satisfied: ${ }^{[63 !}$

$V_{2}>2 V_{1}$

Thus, the existence of a band gap for a noncrystalline solid can be understood in terms of chemical concepts.

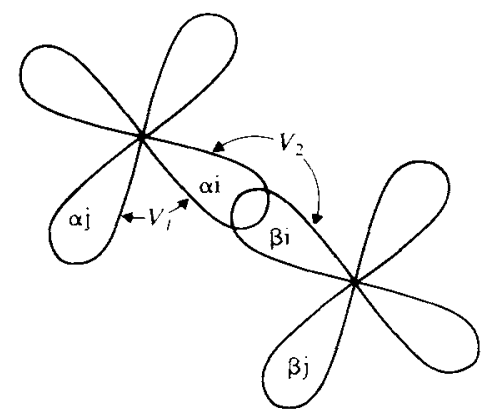

Fig. 13. Weaire-Thorpe model: Intersite $\left(V_{2}\right)$ and intrasite $\left(V_{1}\right)$ interactions.

There are two further consequences of the unimportance of $k$ in disordered materials which are worth mentioning here. The first is that electron (or phonon) states cannot be described in terms of a "band structure", i.e. $E$ vs $k$ (or equivalently a dispersion curve, $\omega$ vs $q$ ), but instead the "density of states" is the only parameter which equally well applies to an amorphous material as to a crystalline solid. (See Figure 14 for a schematic representation of the form of the density of electron states for an amorphous semiconductor.) The second ramification of the fact that $k$ is no longer a good quantum number is that optical spectra are affected. In crystals, periodicity dictates that the wave vector of the initial and final states involved in the transition is conserved; no such restriction applies for the amorphous case and as a result the spectra of amorphous materials do not exhibit the sharp features characteristic of crystals and which are due to critical points in the joint density of states (i.e. when $\nabla_{\mathrm{k}} E=0$ ) dominating the matrix element for the transition (see Fig. 12).

The other important effect of disorder on electron states is the possibility of localization. Whilst this behavior is not unusual as viewed by a chemist, for whom the concept of localization of electrons in molecular orbitals is commonplace, it is most unusual for those versed in conventional crystalline solid-state theory, such as physicists, for whom the electron states in solids are viewed in terms of essentially free electrons only weakly scattered by the ion cores. One approach proposed by Mott et al. ${ }^{[61]}$ is that in an amorphous material there exists a critical energy below which (for electrons in the conduction band) the electrons are spatially localized in the region of a single atomic site, and above which they are spatially extended throughout the material, as in a crystal, although the phases of the wave function may be random from site to site. This picture is indicated in Figure 14, and the critical energies $E_{c}$ in the conduction band and $E_{\mathrm{v}}$ in the valence band are often termed "mobility edges". This is because electrons in extended states, say just above $E_{\mathrm{c}}$, do not require thermal energy for transport in the bands (their motion being diffusive) and consequently they have relatively high mobilities $\left(\mu \approx 10 \mathrm{~cm}^{2} \mathrm{~V}^{-1} \mathrm{~s}^{-1}\right)$, whereas localized electrons can only move from site to site via interactions with phonons and consequently their mobility is activated and much smaller $\left(\mu \simeq 10^{-2} \mathrm{~cm}^{2} \mathrm{~V}^{-1} \mathrm{~s}^{-1}\right)$; the thousandfold change in mobility (and hence conductivity) at $E_{\mathrm{c}}$ and $E_{\mathrm{v}}$ means that $\left(E_{\mathrm{c}}-E_{\mathrm{v}}\right)$ marks a "mobility gap" even though the density of (localized) electron states may well extend considerably 
into this region and be continuous through $E_{\mathrm{c}}$ and $E_{\mathrm{v}}$ (see Fig. 14).

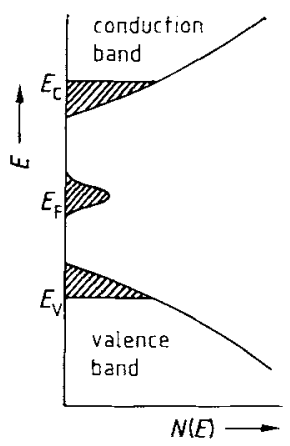

Fig. 14. Schematic density of states, $N(E)$, for an amorphous semiconductor. The mobility edges at $E_{\mathrm{c}}$ and $E_{\mathrm{v}}$ are shown, and all states lying in energy between these levels are local. ized (and shown shaded). A band of states near the Fermi level, $E_{\mathrm{F}}$, due to defects, is also shown.

It should be mentioned in passing that structural effects such as dangling bonds have electron states which lie approximately in the middle of the gap, near the Fermi energy, $E_{\mathrm{F}}$. Electrical conduction by means of transport amongst these levels can only proceed by a process of phonon-assisted tunneling, in which the probability of transfer between two sites separated in distance by $R$ and in energy by $W$ (see Fig. 15) is given by

$p=v_{\mathrm{ph}} \exp (-2 \alpha R) \exp (-W / k T)$

where $v_{\mathrm{ph}}$ is a typical phonon frequency. The first exponential factor represents the tunneling probability between the two sites having localized electron wave functions associated with them of the form $\psi \propto \exp (-\alpha r)$, and the second factor represents the probability of a phonon existing having an energy $W$. At very low temperatures, the proba-

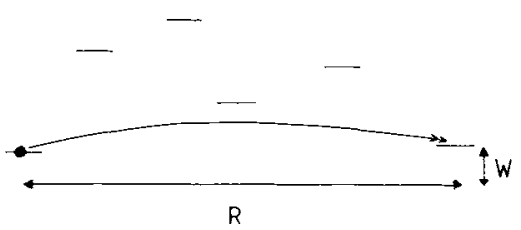

Fig. 15. Schematic diagram of the variable-range hopping process for electron transport among defect electron energy leveis near the Fermi level at low temperatures.

bility of electron transfer can be optimized by tunneling, not to the nearest site but to a more distant site which has a smaller energy difference, $W$. Optimization of this sort led Mott to derive his famous law for "variable-range hopping" [Eq. (8)] where the factor $A$ is defined by Equation (9)

$\sigma=\sigma_{0} \exp \left(-A T^{-1 / 4}\right)$

$A=2.1\left[\alpha^{3} / k N\left(E_{\mathrm{F}}\right)\right]^{1 / 4}$

and where $N\left(E_{\mathrm{F}}\right)$ is the density of states at the Fermi level. Such behavior has been observed many times in various amorphous semiconductors, and an example $e^{[64]}$ for the case of a-silicon is shown in Figure 16. An interesting effect is observed when the conductivity is measured in gap geometry and the thickness of a film is progressively reduced: when the thickness of the film, $d$, becomes comparable to the hopping distance, the transport process be-
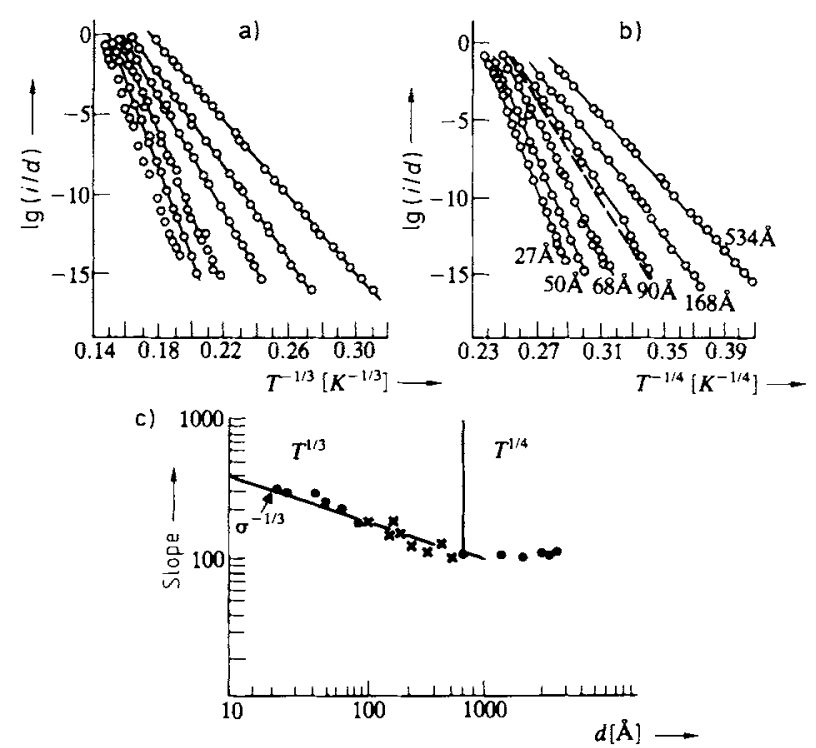

Fig. 16. Variable-range hopping conduction in a-Si; a) two-dimensional transport exhibiting $T^{-1 / 3}$ behavior; b) three-dimensional transport exhibiting $T^{-1 / 4}$ behavior; $i / d$ is the quotient of the current strength and thickness of the conducting layer (in $\AA$ ) ; c) dependence of the exponents on the thickness $d$.

comes essentially two-dimensional and a different temperature dependence of the conductivity is predicted in accordance with Equation (10), where the constant $B$ becomes thickness dependent [Eq. (11)]:

$\sigma_{2 \mathrm{D}}=\sigma_{0}^{\prime} \exp \left(-B T^{-1 / 3}\right)$

$B=\left[3 \alpha^{2} / d k N\left(E_{\mathrm{F}}\right)\right]^{1 / 3}$

This picture of a well-defined mobility edge, with electrical conduction at high temperatures taking place by means of electron transport in the bands of extended states beyond the mobility edges, has not gone unchallenged however. A rival theory for electrical conduction has been proposed $^{[62]}$, particularly for the case of chalcogenide glasses (alloys of $\mathrm{Ge}$, As etc. containing $\mathrm{S}$ and $\mathrm{Se}$ ), in terms of "polaron" transport. A polaron is a charge carrier which causes a considerable amount of lattice distortion when it occupies a site. As a result the charge carrier digs itself a potential well; it must carry the lattice distortion along with it when it moves, and hence the mobility is thermally activated. Polarons can be of several types (see Fig. 17): "electrostatic" polarons can exist in ionic solids, where the lattice distortion occurs because of the electrostatic interaction between the ions of the lattice and the charge carrier; "molecular" polarons exist when the interaction is of a covalent type involving some change in the bonding between atoms when a charge carrier is inserted between them, and such polarons are found in solid rare gases and perhaps in chalcogenide glasses, where a hole formed in a chalcogen $p-\pi$ orbital (forming the top of the valence band) can result in a local distortion. The essential differences between the polaron model and the band model for electronic conduction in amorphous semiconductors can be illustrated by reference to the defining equation for the conductivity, $\sigma$ [Eq. (12)]

$\sigma=n e \mu$ 

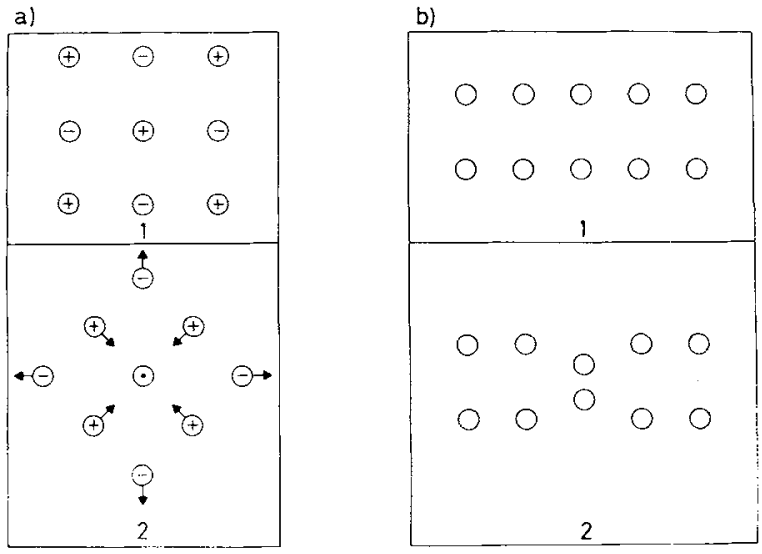

Fig. 17. Schematic representation: (a) electrostatic and (b) molecular polarons. 1: Undisturbed lattice; 2 : effects of the polarons.

where $n$ is the charge-carrier density, $e$ the electronic charge, and $\mu$ the mobility. The band model assumes that $\mu$ is high and temperature independent (transport in extended states) and that $n$ is small and thermally activated (carriers must be thermally excited across the band gap), whereas the polaron model assumes that $\mu$ is small and thermally activated (because transport involves interaction with phonons) and that $n$ is large and temperature independent (because every atomic site can form a polaron center). It is still not clear which model is the more appropriate.

The other challenge to Mott's picture of the electronic structure of noncrystalline solids has been to the concept of the mobility edge. Recent studies ${ }^{[65]}$ of localization using so-called scaling methods (similar to those employed in studies of phase-transition phenomena) have indicated that the mobility and conductivity change in a continuous,

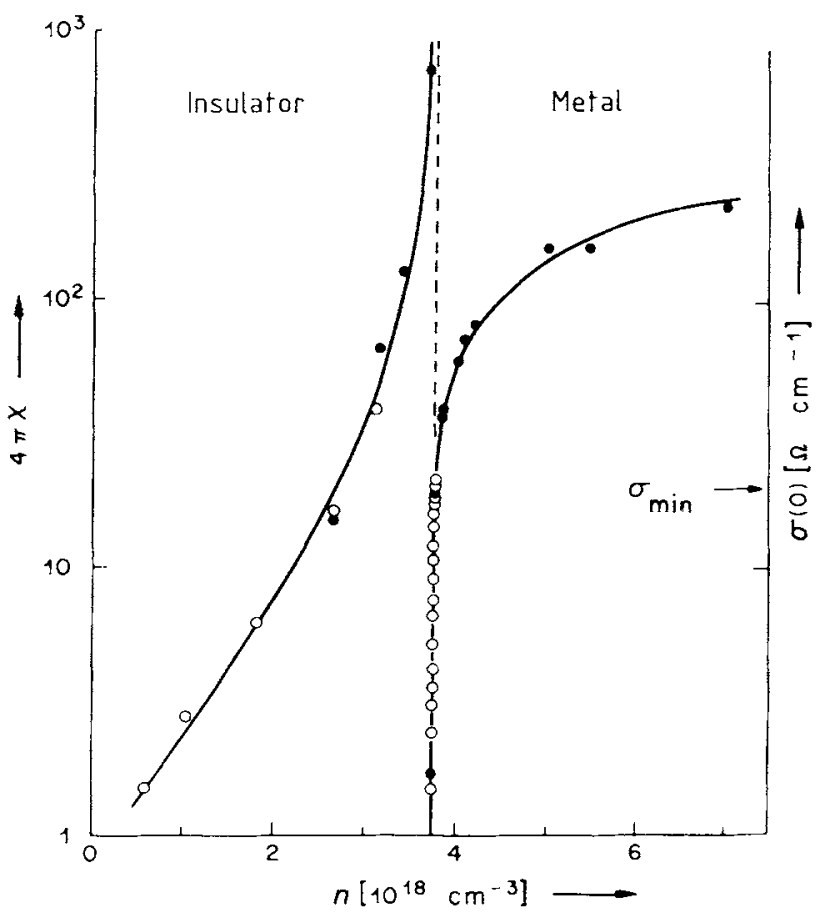

Fig. 18. Crystalline phosphorus-doped silicon; behavior of the conductivity, $\sigma$, and the susceptibility, $\chi$, as a function of the charge carrier density $n$ near the localization edge for electrons. rather than a discontinuous, manner through the localization transition. Experimental support ${ }^{[66]}$ for this picture has come from studies of crystalline phosphorus-doped silicon (Si : P), where the randomly distributed $\mathrm{P}$ atoms act as donors, and the electrons experience a disorder potential due to the random positions of the $\mathrm{P}^{+}$ions; Figure 18 shows the behavior observed. It is interesting to note from this figure that values of the conductivity are measured below the value of the "minimum metallic conductivity", $\sigma_{\min } \simeq 500 \Omega^{-1} \mathrm{~cm}^{-1}$, proposed by Mott as the limiting conductivity of a disordered material on approaching the localization transition (i.e. $E=E_{c}$; cf. Fig. 14) from the metallic side.

\section{Heterogeneous Catalysis and the Noncrystalline State}

It has long been recognized that periodicity in a crystallographic sense, though often a vital and generally desirable attribute of most heterogeneous catalysts, is by no means essential. Indeed there are well-known molten salt catalysts which, by definition, lack long-range order. One of these, molten $\mathrm{ZnCl}_{2}$, was used over a century ago by $L a$ Bel et al. ${ }^{67]}$ to synthesize hexamethylbenzene in a 'onestep' conversion of methanol.

$\mathrm{CH}_{3} \mathrm{OH} \longrightarrow \mathrm{C}_{6}\left(\mathrm{CH}_{3}\right)_{6}+\mathrm{H}_{2} \mathrm{O}$.

Perhaps the most widely known and arguably the most widely used catalysts until the advent of zeolitic catalysts in the petrochemicals industry in the mid sixties, are the silica-alumina 'gels', consisting usually of about 10 to 25 per cent $\mathrm{Al}_{2} \mathrm{O}_{3}$ in solid-solution in ' $\mathrm{X}$-ray amorphous' silica $\left(\mathrm{SiO}_{2}\right)$. This composition range has advantageous Bronsted acidity attributable to the presence of accessible groups of the type,<smiles></smiles>

from which the proton is rather readily detached by a conjugate base, such as an olefin. Such Brønsted acidity, coupled with the very large surface areas (typically 200-800 $\mathrm{m}^{2} \mathrm{~g}^{-1}$ ) is the root cause of the catalytic activity of amorphous $\mathrm{SiO}_{2}-\mathrm{Al}_{2} \mathrm{O}_{3}$ materials and gives rise to their widespread use in isomerization, hydration, and cracking of hydrocarbons.

There has been much speculation as to whether supported catalysts are amorphous or whether they possess crystallographic order, and indeed persuasive arguments have recently been formulated suggesting that a close epitactic match between a crystalline support (the substratum) and an oriented monolayer (supported) active catalyst phase is a prerequisite for the performance of oxide catalysts for the selective oxidation of hydrocarbons. To be specific, supported $\mathrm{V}_{2} \mathrm{O}_{5}$ catalysts on the anatase polymorph of $\mathrm{TiO}_{2}$ are of considerable commercial importance, ${ }^{[9]}$ since they exhibit high activity and selectivity in the oxidative conversions of $o$-xylene to phthalic anhydride, a valuable precursor in the production of anthraquinone and polyesters. 


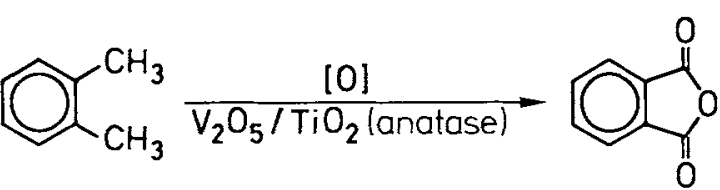

$\left(\mathrm{V}_{2} \mathrm{O}_{5}\right.$-alone or when supported on other substrates - is a rather indifferent catalyst for this reaction.)

EXAFS and XANES (X-ray absorption near edge structure) investigations on typical $\mathrm{V}_{2} \mathrm{O}_{5} / \mathrm{TiO}_{2}$ catalysts and a range of related compounds leave us in little doubt that the catalytically active (monolayer) phase of $\mathrm{V}_{2} \mathrm{O}_{5}$ supported on anatase is not in epitactic registry with the substratum but is in a state of structural disorder. The experimental facts and their interpretation are briefly as follows. In Figure 19 we show the EXAFS spectra, beyond the K-edge of

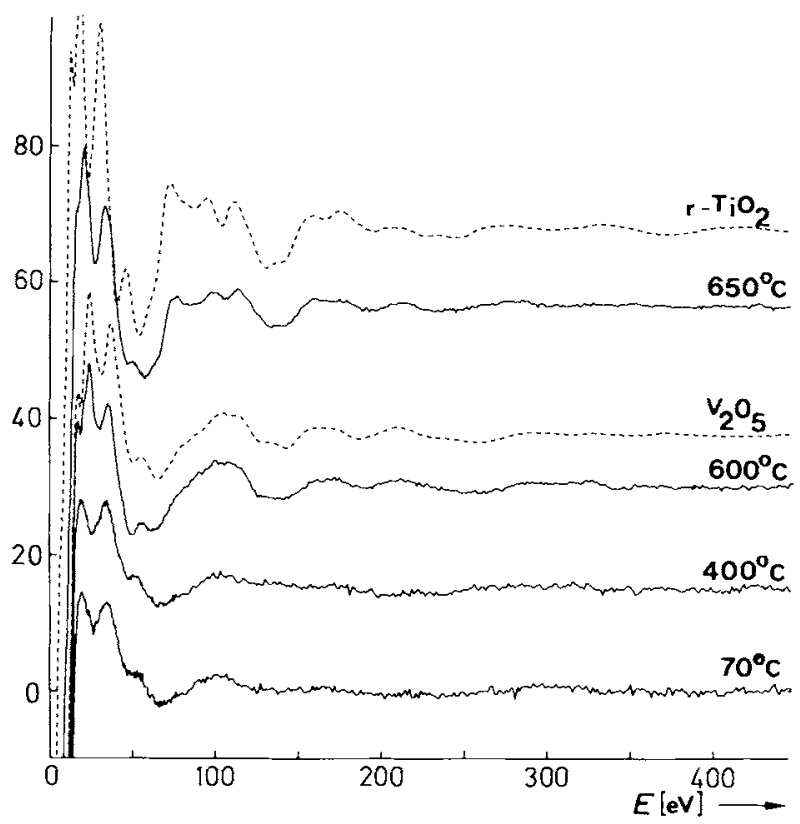

Fig. 19. EXAFS spectra for the vanadium edge of the $\mathrm{V}_{2} \mathrm{O}_{5}$ monolayer on $\mathrm{NOO}_{2}$ (anatase) after $5 \mathrm{~h}$ heat treatments at various temperatures: EXAFS spectra of crystalline $\mathrm{V}_{2} \mathrm{O}_{5}$ and $\mathrm{TiO}_{2}$ (rutile) shown as dashed curve. $E$ denotes the energy above the absorption edge of vanadium. The numbers on the ordinates denote the EXAFS amplitude.

vanadium $\left(\mathrm{Z}=23\right.$ ), for a typical $\mathrm{V}_{2} \mathrm{O}_{5} / \mathrm{TiO}_{2}$ (anatase) catalyst after heat treatment at various temperatures; also shown are the EXAFS spectra of crystalline $\mathrm{V}_{2} \mathrm{O}_{5}$ and of $\mathrm{TiO}_{2}$ rutile (beyond the $\mathrm{K}$-edge of titanium $(\mathrm{Z}=22)$ ). It is clear that the bottom two EXAFS curves in Figure 19 are essentially the same and possess poor structure, characteristic of a poorly crystalline or amorphous phase. Few or no structural changes occur upon heating the system to $350^{\circ} \mathrm{C}$, which is not far removed from the optimal temperature for catalytic conversion. On heating beyond $600^{\circ} \mathrm{C}$, we know, from X-ray diffraction and microcalorimetry, that the finely dispersed anatase sinters and the vanadium oxide is liberated. Clearly the jettisoned oxide is, from its EXAFS spectrum, indistinguishable from crystalline $\mathrm{V}_{2} \mathrm{O}_{5}$. Upon yet further heating (to $650^{\circ} \mathrm{C}$ ) some oxygen is expelled and a solid-solution, $\mathrm{Ti}_{1-\mathrm{x}} \mathrm{V}_{\mathrm{x}} \mathrm{O}_{4}$, forms, possessing a rutile structure (as indicated by the top two curves in Figure 19). XANES studies fully confirm this picture. More- over, a quantitative treatment of the EXAFS data yields further insight into the basic structural unit of the catalytically active, disordered phase: it has two terminal $\mathrm{V}_{-} \mathrm{O}$ bonds of length $1.65 \AA$ and two bridging V-O-Ti bonds in which the $\mathrm{V}$-O distance is $1.90 \AA$.

It would be premature to draw too many conclusions from the $\mathrm{V}_{2} \mathrm{O}_{5} / \mathrm{TiO}_{2}$ (anatase) system. Until such time as EXAFS data for a range of temperatures (under in situ conditions) become available it is difficult to decide whether the intrinsic disorder in the active catalyst is of a static or dynamic kind. Moreover, we do not yet know how generally valid is the picture of noncrystallinity for supported monolayers of catalytically active materials anchored to crystalline substrate.

\section{Materials Applications and Design}

A variety of materials of vital technological importance are amorphous or quasi-crystalline. For instance, a promising photovoltaic material for conversion of solar energy into electricity is amorphous hydrogenated silicon. Magnetic, electric, and other properties of materials rendered amorphous promise several applications as well. The ease of fabrication of glassy or amorphous materials makes them attractive for use in a variety of situations. Thus, there are available glasses which are transparent in most regions of the electromagnetic spectrum, glasses which are semiconducting, glasses which behave like ferroelectrics, and glasses with controlled magnetic behavior. Ferroelectric glasses (such as $\mathrm{LiNbO}_{3}$ ) and glasses with controlled anisotropy of properties are materials worthy of further exploration. We shall deal here, however, with fast-ion conducting glasses and fibers for optical communication in some detail.

One of the most exciting current applications of a glass is the replacement of copper telephone cables by optical fiber light-guides. Light, in either a single mode or a multimode, propagates down the fiber by a process of total internal reflection, a process which occurs whenever light from a high refractive index medium strikes the interface between it and a low index medium with greater than a critical angle of incidence.

Obviously for long-distance applications, the glass fiber must be extremely transparent, i.e. have a very low light loss. There are two possible sources of light loss, scattering and absorption. Scattering (from density fluctuations) follows the Rayleigh law, i.e. the scattered intensity is proportional to $\lambda^{-4}$, where $\lambda$ is the wavelength of the propagating light. There are two possible absorption mechanisms, depending on the wavelength of the light. At short wavelengths, the light can cause electronic excitation across the forbidden energy gap of the insulating glass: the spectral dependence of this absorption process follows the socalled Urbach law, viz. $\alpha=A \exp (B / \lambda)$, i.e. the absorption band has an exponential edge tailing into the gap, and for silica glass, this edge lies in the hard UV region. At the other end of the wavelength scale, i.e. in the IR region, the absorption of light arises from excitation of phonon (vibrational) modes in the glass, and this too has an exponential spectral dependence, viz. $\alpha=C \exp (-D / \lambda)$. The resulting 
wavelength dependence of the overall optical loss in a glass thus has a characteristic V-shaped profile, as shown in Figure 20. Light losses due to Rayleigh scattering are obviously minimized for light of long wavelengths. The low-loss "window" is then given by the intersection of the Rayleigh curve with the multiphonon absorption edge, which for $\mathrm{SiO}_{2}$ occurs at about $1.6 \mu \mathrm{m}$, with a theoretical loss of only $\sim 0.2 \mathrm{~dB} / \mathrm{km}$, although actual fibers are found to have losses near $10 \mathrm{~dB} / \mathrm{km}$, resulting principally from absorption by $\mathrm{OH}$ groups, a ubiquitous impurity.

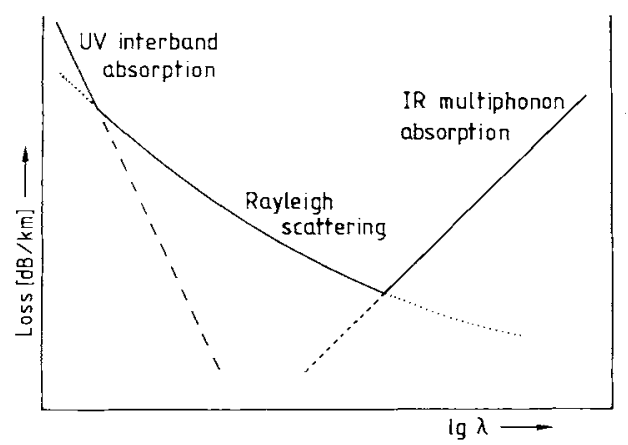

Fig. 20. Mechanisms of light loss in optical tibers as a function of the wavelength $\lambda$.

Since Rayleigh scattering decreases with increasing wavelength, losses can be further reduced by making fibers out of materials for which the multiphonon edge lies at a longer wavelength than for $\mathrm{SiO}_{2}$, and hence intersects the Rayleigh curve at a longer wavelength and a lower value of loss (see Fig. 20). The frequency of phonon modes in a glass can be "tailored" either by choosing a material with a weaker force constant or containing heavier atoms than $\mathrm{SiO}_{2}$. An example of the former strategy is to use fluoride glasses based on $\mathrm{ZrF}_{4}$ (containing other fluorides such as $\mathrm{LaF}_{3}$ and $\mathrm{BaF}_{2}$ as well), where the fluorine atoms are for the most part twofold coordinated in the structure (like the oxygen atoms in $\mathrm{SiO}_{2}$ ), but the bonding between cation and anion is weaker. Whilst these glass materials have extremely low theoretical losses $\left(\simeq 10^{-3} \mathrm{db} / \mathrm{km}\right)$, they are difficult to make and to form fibers from without crystallization taking place. The other alternative, namely substituting light ( $\mathrm{Si}$ ) atoms by heavier ones (e.g. Ge or $\mathrm{Sb}$ ) has also been tried and losses of about $5 \mathrm{db} / \mathrm{km}$ have been achieved for $\mathrm{GeO}_{2}-\mathrm{Sb}_{2} \mathrm{O}_{3}$ alloy glasses.

There is a large and growing number of solids in which certain ions exhibit unusually rapid transport. Such materials associated with high ionic conductivity and small activation energies for ion migration are called fast ion conductors. ${ }^{[68]}$ Typical materials exhibiting fast ion conduction (FIC) are iodides related to AgI and oxides of the type $\mathrm{Zr}_{1-x} \mathrm{Ca}_{x} \mathrm{O}_{2-\mathrm{x}}$ and $\mathrm{Na}_{1-x} \mathrm{Al}_{11} \mathrm{O}_{17+\mathrm{x} / 2}$ ( $\beta$-alumina). Much of the early work on fast ion conduction was related to crystalline materials, but many fast ion conducting glasses have been discovered in recent years. Both $\mathrm{Li}^{+}$and $\mathrm{Ag}^{+}$ conducting glasses are known. $\mathrm{AgI}$ is almost always a component of the $\mathrm{Ag}^{+}$-containing FIC's; $\mathrm{Li}^{+}$FIC's are generally formed in systems with network structures. The ionic conductivity of these fast ion conducting glasses ${ }^{[69]}$ is comparable to those of crystalline FIC's (Fig. 21). The glass

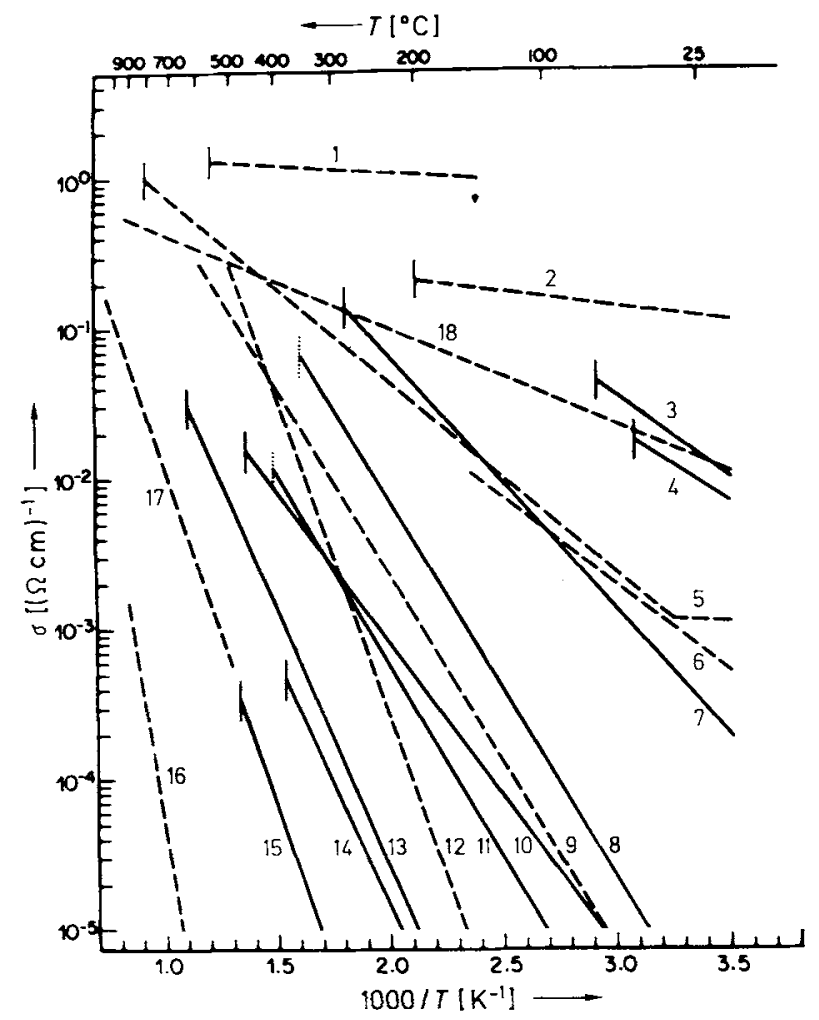

Fig. 21. Ionic conductivities of at variety of amorphous, 1 and crystalline (--) superionic conductors as a function of the temperature [69]. Vertical lines: $T_{\mathrm{g}}$ and $T_{\mathrm{f}}$, respectively (see Fig. 2); vertical dotted lines: $T_{\mathrm{g}}$, estimated. $\mathrm{I}: \alpha-\mathrm{AgI} ; 2: \mathrm{RbAg}_{4} \mathrm{I}_{5} ; 3: 29.8 \mathrm{Ag}_{2} \mathrm{O}-40.4(\mathrm{Agl})_{2}-29.8 \mathrm{P}_{2} \mathrm{O}_{3}: 4: 28.6 \mathrm{Ag}_{2} \mathrm{O}-$ $42.8(\mathrm{Agl})_{2}-28.6 \mathrm{MoO}_{3} ; 5: \mathrm{Li}_{3} \mathrm{~N}: 6: \beta-\mathrm{PbF}_{2}-2 \% \mathrm{KF} ; 7: 60 \mathrm{Ag}_{2} \mathrm{~S}-5 \mathrm{GeS}-$ $45 \mathrm{GeS}_{2} ; 8: 35 \mathrm{Li}_{2} \mathrm{O}-30 \mathrm{Li}_{2} \mathrm{SO}_{4}-10(\mathrm{LiCl})_{2}-12.5 \mathrm{SiO}_{2}-12.5 \mathrm{~B}_{2} \mathrm{O}_{3}: 9: \mathrm{Li}_{4} \mathrm{~B}_{7} \mathrm{O}_{12} \mathrm{Cl}$ 10: $\mathrm{Li}_{2} \mathrm{O}-\mathrm{Nb}_{2} \mathrm{O}_{5}\left(\mathrm{LiNbO}_{3}\right) ; 11: 26.9 \mathrm{Li}_{2} \mathrm{O}-9.0(\mathrm{LiCl})_{2}-64.1 \mathrm{~B}_{2} \mathrm{O}_{3}\left(\mathrm{Li}_{4} \mathrm{~B}_{7} \mathrm{O}_{32} \mathrm{Cl}\right)$ 12: $\mathrm{LiAlSiO}_{4}$; 13: $25 \mathrm{Li}_{2} \mathrm{O}-25 \mathrm{Al}_{2} \mathrm{O}_{3}-50 \mathrm{SiO}_{2}\left(\mathrm{LiAlSiO}_{4}\right) ; \quad 14$ $\mathrm{Z}_{0.6} \mathrm{Ba}_{(3.33} \mathrm{Y}_{0,07} \mathrm{~F}_{3,27} ; 15: 25 \mathrm{Li}_{2} \mathrm{O}-75 \mathrm{~B}_{2} \mathrm{O}_{3} ; 16: \mathrm{LiNbO}_{3} ; 17: \mathrm{ZrO}_{2}-9 \% \mathrm{Y}_{2} \mathrm{O}_{3}$; 18: $\mathrm{NaAl}_{11} \mathrm{O}_{17}(\beta$-alumina).

FIC's offer several advantages for their use in power devices and related systems, besides the ease of fabrication. Properties of glassy materials can be manipulated through composition control. The isotropic behavior of glasses can also be of considerable advantage.

Received: September 20, 1985 [A 562 IE] German version: Angew. Chem. 98 (1986) 31

[1] S. R. Elliott: Physics of Amorphous Materials. Longman, London 1984.

[2] D. Shechtman, 1. Bleck, D. Gratias, J. W. Cahn, Phys. Rev. Lett. 53 (1984) 1951.

[3] D. Levine, P. J. Steinhardt, Phys. Rev. Lett. 53 (1984) 2477.

[4] D. R. Nelson, B. I. Halperin, Science 229 (1985) 233; cf. also L. Pauling, Nature (London) 317 (1985) 512

[5] R. Penrose, Bull. Inst. Math. Appl. 10 (1974) 266.

[6] A. L. Mackay, Physica II4A (1982) 609; Sov. Phys. Crystallogr. 26 (1981) 517.

[7] R. Zallen: The Physics of Amorphous Solids, Wiley, New York 1983.

[8] J. M. Thomas, Proc. 8th Int. Congress on Catalysts, Berlin 1984, Vol. I. Verlag Chemie, Weinheim 1984, p. 31; Pure Appl. Chem., in press.

[9] R. Kozlowski, R. F. Pettifer, J. Chem. Soc. Chem. Commun. 1983, 438; J Phys. Chem. 87 (1983) 5178.

[10] R. Elermann, G. M. Parkinson, H. Bässler, J. M. Thomas, J. Phys. Chem 86 (1982) $313 ; 87$ (1983) 544.

[1 I] J. M. Thomas, Ultramicroscopy 8 (1982) 13.

[12] X. L. Yeh, K. Samwer, W. L. Johnson, Appl. Phys. Lett. 42 (1983) 242.

[13] R. Parthasarathy, K. J. Rao, C. N. R. Rao, Chem. Soc. Rev. 12 (1983) 361 .

[14] J. Wong, C. A. Angell: Glass Structure by Spectroscopy, Marcel Dekker, New York 1976.

[15] M. H. Cohen, G. S. Grest, Adv. Chem. Phys. 48 (1981) 455. 
[16] 3. H. Gibbs in J. D. Mackenzie (Ed.): Modern Aspects of the Vitrerus State. Butterworths, London 1963.

[17] H. Kanno, J. Non-Cryst. Solids 37 (1983) 203

[18] M. R. Hoare, J. A. Barker in P. H. Gaskell (Ed.): The Structure of NonCrystalline Materials. Taylor and Francis, London 1977.

[19] P. H. Gaskeli, D. J. Smith, C. J. D. Catto, J. R. O. Cleaver, Nature (London) 281 (1979) 465; L. A. Bursill, J. M. Thomas, K. J. Rao, ibid. 289 (1981) 159 .

[20] K. J. Rao, C. N. R. Rao, Mater. Res. Bull. 13 (1982) 1337.

[21] H. Suga, S. Seki, Faraday Discuss. Chem. Soc. 69 (1980) 221; R. Parthasarathy, K. J. Rao, C. N. R. Rao, J. Phys. Chem. 88 (1984) 49.

[22] S. Bhaltachanya, S. R. Nagel, L. Fleishman, S. Susman, Phys. Rev. Lett. 46 (1984) 1266; U. T. Höchli, H. E. Weibel, L. A. Botner, J. Phys. C 12 (1979) L563.

[23] G. P. Johari, J. W. Goodby, J. Chem. Phys. 77 (1982) 565; G. P. Johari, ibid. 77 (1982) 4619.

[24] C. A. Angell, J. H. R. Clarke, L. V. Woodcock, Adv. Chem. Phys. 48 (1981) 397; L. Woodcock, C. A. Angeil, Phys. Rev. Lett. 47 (1980) 1129.

[25] C. A. Angell in J. M. Honig, C. N. R. Rao (Eds.): Preparation and Characterization of Materiais, Academic Press, New York 1981.

[26] M. S. Sceats, S. A. Rice in F. Franks (Eds.): Water, A Comprehensive Treatise, Vol. 7, 1982.

[27] J. A. McMillan, S. C. Los, J. Chem. Phy's. 42 (1965) 529

[28] C. A. Angell, E. J. Sare, J. Chem. Phys. 52 (1970) 1055.

[29] M. Sugisaki, H. Sugan, S. Seki, J. Chem. Soc. Jpn. 4 (1968) 2591.

[30] P. Brugellen, E. Mayer, Nature (London) 288 (1980) 569, 579.

[31] J. Dubochet, J. Lepault, R. Freeman, J. Microse. (Oxford) 128 (1982) 219.

[32] D. R. MacFarlane, C. A. Angell, J. Phys. Chem. 88 (1984) 759

[33] R. J. Temkin, G. A. N. Connell, W. Paul, Adv. Phys. 22 (1973) 581

[34] E. A. Stern, Phys. Rev. Bll (1974) 4836; B. K. Teo, Acc. Chem. Res. 13 (1980) 412

[35] D. R. Sandstrom, F. W. Lytle, Annu. Rev. Phys. Chem. 30 (1979) 215.

[36] R. Parthasarathy, P. R. Sarode, K. J. Rao, C. N. R. Rao, Proc. Indian Nat. Sci. Acad.. Part A 48 (1982) 119.

[37] J. Wong, Top. Appl. Phys. 46 (1981).

[38] B. G. Williams, G. M. Parkinson, T. G. Sparrow, C. J. Eckhardt, J. M. Thomas, Chem. Phys. Lett. 78 (1981) 434.

[39] B. G. Williams, J. M. Thomas, Int. Rev. Phys. Chem. 3 (1983) 39.

[40] B. G. Williams, T. G. Sparrow, J. M. Thomas, J. Chem. Soc. Chem. Commun. 1983, 1434.

[41] E. R. Andrew, Int. Rev. Phys. Chem. I (1981) 195

[42] E. Lippmaa, M. Alla, T. Juherm, Proc 19th Ampere Congress Heidelberg 1976, p. 113.
[43] P. J. Bray in L. D. Pye, V. D. Frechette, N. J. Kreid] (Eds.): Borate Glasses: Structure, Properties. Applications, Plenum, New York 1978.

[44] C. A. Fyfe, J. M. Thomas, J. Klinowski, G. C. Gobbi, Angew. Chem. 95 (1983) 257: Angew. Chem. Int. Ed. Engl. 22 (1983) 259: J. M. Thomas, J. Klinowski, P. A. Wright, R. Roy, ibid. 95 (1983) 643 and 22 (1983) 614.

[45] J. M. Thomas, J. Klinowski, S. Ramdas, B. K. Hunter, D. T. B. Tennakoon, Chem. Phys. Lett. 102 (1983) 148; S. Ramdas, J. Klinowski, Natu4e (London) 308 (1984) 521 .

[46] R. Dupree, R. F. Pettifer, Nature (London) 308 (1984) 523

[47] W. H. Zachariasen, J. Am. Chem. Soc. 54 (1932) 3841.

[48] D. E. Polk, J. Non-Cryst. Solids 5 (1971) 365.

[49] L. A. Bursill, J. M. Thomas, J. Phys. Chem. 85 (1981) 3007.

[50] J. M. Thomas, L. A. Bursill, Angew. Chem. 92 (1980) 755; Angew. Chem. Int. Ed. Engl. 19 (1980) 745.

[51] L. A. Bursill, J. M. Thomas, K. J. Rao, Nature (London) 289 (1981) 157.

[52] J. D. Bernal, Proc. R. Soc. London Ser. A 280 (1964) 299

[53] P. H. Gaskelt, J. Non-Cryst. Solids 32 (1979) 207.

[54] C. N. R. Rao, Chem. Scr. 19 (1982) 124.

[55] A. Reller, D. A. Jefferson, J. M. Thomas, M. K. Uppal, J. Phys. Chem. 87 (1983) 913

[56] A. Reller, J. M. Thomas, D. A. Jefferson, M. K. Uppal, Proc. R. Soc. London, in press.

[57] T. G. Fowler, S. R. Elliott, J. Non-Cryst. Solids 59-60 (1983) 957.

[58] B. G. Sibernagel, M. S. Whittingham, J. Chem. Phys. 64 (1976) 3670.

[59] T. G. Fowier, Ph. D. Thesis, University of Cambridge 1984.

[60] S. R. Elliott, J. Non-Cryst. Solids 59-60 (1983) 899.

[61] N. F. Mott, E. A. Davis: Electronic Processes in Non-Crystalline Materiats, 1979.

[62] D. Emin in P. G. Le Comber, J. Mort (Eds.): Electronic and Siructural Properties of Amorphous Semiconductors, Academic Press, New York 1973.

[63] D. Weaire, M. F. Thorpe, Phys. Rev. B4 (1971) 2508, 3518.

[64] M. L. Knotek in M. G. Brodsky, S. Kirkpatrick, D. Weaire (Eds.): Proc. int. Conf. on Tetrahedrally Bonded Amorphous Semiconductors, Vol 20. 1974 , p. 297.

[65] E. Abrahams, P. W. Anderson, D. C. Liccardello, T. V. Ramakrishnan, Phys. Rev. Lett. 42 (1979) 673.

[66] H. F. Hess, K. De Londe, T. F. Rosenbaum, G. A. Thomas, Phys. Rev. $B 25$ (1982) 5578 .

[67] J. A. Le Bel, W. H. Greene, Am. Chem. J. 2 (1880) 20.

[68] P. Hagenmuller, W. Van Gool (Eds.): Solid Electrolytes. Academic Press, New York 1978

[69] H. L. Tuller, D. P. Button, D. R. Uhlmann, J. Non-Cryst. Solids 40 (1980) 93 . 